\title{
Deficient LRRC8A-dependent volume- regulated anion channel activity is associated with male infertility in mice
}

\author{
Jianqiang Bao, ${ }^{1}$ Carlos J. Perez, ${ }^{1}$ Jeesun Kim, ${ }^{1}$ Huan Zhang, ${ }^{2}$ Caitlin J. Murphy, ${ }^{3}$ Tewfik Hamidi, \\ Jean Jaubert, ${ }^{4}$ Craig D. Platt, ${ }^{5}$ Janet Chou, ${ }^{5}$ Meichun Deng, ${ }^{6}$ Meng-Hua Zhou, ${ }^{6}$ Yuying Huang, ${ }^{6}$ \\ Héctor Gaitán-Peñas, ${ }^{7,8}$ Jean-Louis Guénet, ${ }^{4}$ Kevin Lin, ${ }^{1}$ Yue Lu, ${ }^{1}$ Taiping Chen, ${ }^{1,9}$ Mark T. Bedford,, \\ Sharon Y.R. Dent,, ${ }^{1,9}$ John H. Richburg, ${ }^{3}$ Raúl Estévez, ${ }^{7,8}$ Hui-Lin Pan, ${ }^{6}$ Raif S. Geha, ${ }^{5}$ Qinghua Shi, ${ }^{2}$ \\ and Fernando Benavides ${ }^{1,9}$ \\ 'Department of Epigenetics and Molecular Carcinogenesis, The University of Texas MD Anderson Cancer Center, Smithville, \\ Texas, USA. ${ }^{2}$ School of Life Science, University of Science and Technology of China, Hefei, China. ${ }^{3}$ The University of Texas \\ at Austin, College of Pharmacy, Austin, Texas, USA. ${ }^{4}$ Unité de Génétique de la Souris, Institut Pasteur, Paris, France. \\ ${ }^{5}$ Division of Immunology, Boston Children's Hospital, Boston, Massachusetts, USA. ${ }^{6}$ Center for Neuroscience and Pain \\ Research, Department of Anesthesiology and Perioperative Medicine, The University of Texas MD Anderson Cancer Center \\ Houston, Texas, USA. 'Unitat de Fisiología, Departament de Ciències Fisiològiques, IDIBELL-Institute of Neurosciences, \\ Universitat de Barcelona, L'Hospitalet de Llobregat, Spain. ${ }^{8} \mathrm{U}-750$, CIBERER, ISCIII, Barcelona, Spain. ${ }^{9}$ UTHealth Graduate \\ School of Biomedical Sciences, Houston, Texas, USA.
}

\begin{abstract}
Ion channel-controlled cell volume regulation is of fundamental significance to the physiological function of sperm. In addition to volume regulation, LRRC8A-dependent volume-regulated anion channel (VRAC) activity is involved in cell cycle progression, insulin signaling, and cisplatin resistance. Nevertheless, the contribution of LRRC8A and its dependent VRAC activity in the germ cell lineage remain unknown. By utilizing a spontaneous Lrrc8a mouse mutation (c.1325delTC, p.F443*) and genetically engineered mouse models, we demonstrate that LRRC8A-dependent VRAC activity is essential for male germ cell development and fertility. Lrrc8a-null male germ cells undergo progressive degeneration independent of the apoptotic pathway during postnatal testicular development. Lrrc8a-deficient mouse sperm exhibit multiple morphological abnormalities of the flagella (MMAF), a feature commonly observed in the sperm of infertile human patients. Importantly, we identified a human patient with a rare LRRC8A hypomorphic mutation (c.1634G>A, p.Arg545His) possibly linked to Sertoli cell-only syndrome (SCOS), a male sterility disorder characterized by the loss of germ cells. Thus, LRRC8A is a critical factor required for germ cell development and volume regulation in the mouse, and it might serve as a novel diagnostic and therapeutic target for SCOS patients.
\end{abstract}

Conflict of interest: The authors have declared that no conflict of interest exists.

Submitted: January 11, 2018 Accepted: July 11, 2018 Published: August 23, 2018

\section{Reference information:} JCI Insight. 2018;3(16):e99767. https://doi.org/10.1172/jci. insight.99767.

\section{Introduction}

The cells of the body are frequently exposed to the stress of hyper- and hypotonic microenvironments. Thus, cell volume regulation is of fundamental importance for the physiological function and metabolic homeostasis for almost every vertebrate cell. Vertebrate cells have evolved multiple types of both tissue-specific and ubiquitous plasma membrane channels that counteract cell swelling by decreasing cell volume through regulatory volume decrease (RVD) and cope with shrinking by increasing volume through regulatory volume increase (RVI) (1). Among those volume-regulating channels are the dedicated water channels of aquaporin family proteins (Aqp0-12), specific ion channels $\left(\mathrm{K}^{+}\right.$, $\left.\mathrm{Na}^{+}, \mathrm{Cl}^{-}\right)$, and specialized organic solute transporters $(1,2)$. The transport of inorganic ions and smal organic osmolytes, such as taurine and amino acids, out of the cells is accompanied by efflux of water either through passive diffusion across the cell membrane or through the more efficient aquaporin channel proteins, leading to the recovery of the cell volume and size $(1,3)$. RVD is achieved primarily through chloride channels, i.e., $\mathrm{Cl}^{-}$current, which is often gated by the volume-regulated anion channel (VRAC), which can sense cell swelling or ionic strength (4-7). 
Being able to regulate cell volume in changing osmotic conditions is critically important for the proper development of sperm. In mammals, spermatogenesis ensures continuous sperm production through a tightly orchestrated series of events in the testis.

Upon completion of spermatogenesis, the condensed sperm exit the seminiferous tubules and transit through the rete testis to the efferent ductules, and to the epididymis, where they undergo further functional maturation. Specialized tight junctions between adjacent Sertoli cells in the seminiferous epithelium delineate 2 cellular compartments, the basal compartment and the adluminal compartment, which are pivotal for the differentiation of germ cells inside the seminiferous tubules (8). Unlike blood plasma, which maintains a constant osmolality at $\sim 290 \mathrm{mOsm} / \mathrm{kg}$, the luminal fluids in different segments of the male reproductive tract possess distinct components and, thus, exhibit a highly dynamic range of osmolality. In the mouse, osmolality ranges from $\sim 350 \mathrm{mOsm} / \mathrm{kg}$ in the seminiferous tubules to approximately 290 $\mathrm{mOsm} / \mathrm{kg}$ in the rete testis and to approximately $410 \mathrm{mOsm} / \mathrm{kg}$ in the cauda epididymis (9-11). While the tight junctions create a specialized adluminal milieu to protect spermatogenesis from the insults of the vascular blood contents, both the rete testis and the initial segment of the epididymis (caput) are entirely bathed in the blood vessels with an osmolality similar to that of blood plasma (8). In normal mouse sperm, alterations of the local osmotic microenvironment in the caput can elicit irreversible sperm swelling (12, 13). In addition, sperm naturally encounter a physiological osmotic decline when moving from the male cauda $(\sim 410 \mathrm{mOsm} / \mathrm{kg})$ into the fluid of the female reproductive tract, which has an osmolality similar to the blood plasma $(3,11,14)$. These dynamic osmotic alterations present a significant challenge to germ cell development and survival, and thereby necessitate volume regulation to counteract the osmotic fluctuations $(3,11,12)$. When RVD fails, sperm become swollen with a "coiled" or "angulated" flagellar morphology, which helps them avoid excessive plasma membrane stretching. However, coiled sperm lose their forward motility and, as a consequence, are unable to penetrate the female utero-tubal mucus to fertilize oocytes $(3,11,12)$. In fact, coiled/deformed sperm tails are commonly observed in samples from clinically infertile human patients, and this phenotype is closely associated with male infertility. However, the molecular mechanisms or the causative mutations underlying this phenomenon are poorly understood.

The leucine-rich repeat-containing 8 (LRRC8) family comprises 5 paralogs ( $L R R C 8 A,-B,-C,-D$, and $-E$ ) in the mammalian genomes, which encode proteins with 4 transmembrane helices and 17 leucine-rich repeats (LRRs). They were initially speculated to be involved in cell-to-cell communication and signaling cascades $(15,16)$. Of the LRRC8 family, LRRC8A was the first member to be described, following the discovery of a human patient diagnosed with agammaglobulinemia as a result of a $\mathrm{B}$ cell defect. This disease resulted from a heterozygous translocation causing the deletion of 3 LRRs at the C-terminus of LRRC8A (designated as LRRC8A ${ }^{\Delta 91 /+35}$ ) (17). So far, this is the only causative mutation identified in $L R R C 8 A$ associated with a human disease. Recently, 2 groups independently identified LRRC8 heteromers as the pore-forming subunits of the long-sought VRAC $(18,19)$. In particular, the LRRC8A protein (also known as SWELL1) was validated as the obligatory component of the VRAC $(6,18,19)$ and functions in conjugation with at least 1 other LRRC8 family member in a tissue-specific manner $(5,20,21)$. Subsequent studies, mostly performed in cultured cell lines, showed that LRRC8Adependent VRAC activity is involved in a broad range of biological processes, including cell proliferation, migration, apoptosis, and insulin secretion and uptake, in addition to volume regulation (5, 21-27). We previously reported that the ébouriffé (ebo) mutant mice show male infertility due to secondary structural defects in male germ cells (28). This spontaneous recessive mutation was subsequently identified as a 2-bp deletion in $L r r c 8 a$ that drastically diminishes VRAC activity (29). In contrast to Lrrc8a-KO mice, ebo/ebo mice have normal T cell development and function, suggesting LRRC8A-dependent VRAC activity is dispensable for $\mathrm{T}$ cell development and function $(29,30)$. Nonetheless, the physiological roles and mechanistic insights of LRRC8A during postnatal development are largely unknown.

Here we report that LRRC8A-dependent VRAC function is essential for male germ cell survival and sperm motility. The LRRC8A protein is localized in the membrane of male germ cells at all stages and is most highly abundant in the midpiece of mature sperm tails. In mice, constitutive genetic ablation of $L r r c 8 a$ (KO) led to progressive loss of germ cells (including spermatogonia, spermatocytes, and spermatids) and coiled/deformed sperm tails observed in the cauda epididymis, leading to complete infertility. Germlinespecific conditional Lrrc8-KO (cKO) mice recapitulated the sperm defects observed in the KO mice, suggesting germ cell-autonomous roles of $L r r c 8 a$ in the testis. Importantly, we identified 2 spontaneous mutations that are directly linked to male infertility: the 2-bp deletion ( $\operatorname{rrc} 8 a$, c. $\left.1325 \mathrm{delTG}, \mathrm{p} . \mathrm{F} 443^{*}\right)$ in the $L r r c 8 a^{\text {ebo } / e b o}$ 
mice (29) and a missense mutation in a clinically sterile male patient ( $L R R C 8 A$, c. 1634G > A, p. Arg545His). These studies provide genetic evidence that LRRC8A-dependent VRAC activity is essential for spermatogenesis and suggest that mutations in $L R R C 8 A$ could be examined as a diagnostic target in human patients with coiled/deformed sperm tail morphology.

\section{Results}

LRRC8A is ubiquitously expressed and localizes to the plasma membrane of germ cells in the testis. By analyzing human Genotype-Tissue Expression (GTEx) database (https://www.gtexportal.org), a large collection of RNA sequencing (RNA-seq) datasets from adult organs compiled by the Broad institute (31), we observed that LRRC8A mRNA is ubiquitously expressed in a wide spectrum of human tissues, with modest expression in the testis, comparable with that of spleen and intestine (Figure 1A). Because germline development is strictly time dependent, we examined whether Lrrc8a expression changes during postnatal development in the mouse by analyzing previously published RNA-seq datasets (32). We found that, although Lrrc8e transcripts were not readily detectable in postnatal testis, $L r r c 8 a-d$ were expressed, exhibiting declining expression patterns during postnatal development (Figure 1B). Since the seminiferous tubules of testis contain both Sertoli cells and germ cells at different developmental stages, we determined which cell type predominantly expresses Lrrc8a. By using the centrifugal elutriation approach, we successfully purified 4 types of germ cells as well as Sertoli cells from the mouse testis with purity $>90 \%$ (data not shown) $(33,34)$. Quantitative PCR (qPCR) showed that Lrrc8a mRNA is expressed in both Sertoli cells and germ cells, with the highest expression levels in spermatogonia (Figure 1C). Western blot analysis using testis extracts demonstrated that LRRC8A protein expression declines throughout postnatal development - with the lowest levels at postnatal day 0 (P0) - consistent with the mRNA expression levels (Figure 1D), suggesting that the requirement for LRRC8A varies at different stages of germ cell development.

LRRC8A is a transmembrane protein ubiquitously expressed in a wide range of somatic cell lineages but has not been studied in germ cells. To explore the subcellular localization of LRRC8A, we mechanically separated the adjacent cells in the seminiferous tubules using a "squash preparation" in conjugation with immunofluorescence imaging (35) and found that LRRC8A localizes to the plasma membrane of germ cells, such as the spermatocytes and spermatids (Figure 1E). Intriguingly, immunolabeling on the mature sperm isolated from the cauda epididymis revealed that LRRC8A is predominantly localized in the midpiece segment of mature sperm tails (Figure 1F). This suggests that LRRC8A might be functionally important in both immature germ cells in the testis and mature sperm in the cauda.

The 2-bp frameshift nonsense mutation in the Lrrc8a gene (Lrrc8a, c.1325delTG, p.F443*) is associated with male infertility in the ebo/ebo mice. We became interested in the role of LRRC8A in spermatogenesis when we found that $e b o$ mutant mice, presenting with male infertility, harbored a mutation in the Lrrc $8 a$ gene (28, 29). At that time, we found that sperm from ebo/ebo males displayed severe structural defects (mostly secondary effects as a result of cell swelling), yet the causal relationship between the ebo mutation and aberrant sperm morphology, as well as the molecular mechanisms, were completely unknown. We mapped the $e b o$ locus to proximal mouse chromosome 2 and used targeted next-generation sequencing to discover that the Lrrc8a gene in ebo/ebo mice carries a 2-bp (TG) frameshift deletion in exon 3 (c.1325delTG, p.F443*), introducing a premature stop codon (Supplemental Figure 1A; supplemental material available online with this article; https://doi.org/10.1172/jci.insight.99767DS1) (29). The predicted truncated LRRC8A protein $\left(\mathrm{LRRC} 8 \mathrm{~A}^{\mathrm{F} 443^{*}}\right)$ is presumed to lack the LRR domains from LRR3 to LRR17 but preserves the transmembrane domains (Supplemental Figure 1B). qPCR analysis of testis tissue using a pair of PCR primers designed against the $5^{\prime}$ end of $L r r c 8 a$ mRNA revealed that Lrrc8a mRNA levels in homozygous ebo/ebo $\left(\operatorname{Lrcc} 8 a^{\mathrm{F} 443^{*} / \mathrm{F} 43^{*}}\right)$ mice were reduced to less than one-third of the levels in WT or heterozygous $\left(\operatorname{Lrc} 8 a^{+/ \mathrm{F} 43^{*}}\right)$ mice, suggesting that the mutant transcripts undergo nonsense-mediated mRNA decay (NMD) pathway in the $\operatorname{Lrrc} a^{\mathrm{F} 443^{*} / \mathrm{F} 443^{*}}$ testis (36) (Supplemental Figure 1C). Using an LRRC8A-specific monoclonal antibody generated against the C-terminus of LRRC8A (LRRC8 $\mathrm{A}^{711-810}$ ) that is localized downstream of the premature stop codon, we verified the absence of full-length LRRC8A protein in the $\operatorname{Lrc} 8 \mathrm{a}^{\mathrm{F} 443^{*} / \mathrm{F} 443^{*}}$ testis (Supplemental Figure 1D). However, it is formally possible that the truncated LRRC8A protein exists at low levels in $\operatorname{Lrrc} 8 a^{\mathrm{F} 443^{*} / \mathrm{F} 443^{*}}$ testis.

Multiple morphological abnormalities of the flagella (MMAF) in the sperm of Lrrc8a ${ }^{F 443^{*} / F 443^{*}}$ mice are associated with male infertility. Heterozygous $L r r c 8 a^{+/ F 443^{*}}$ mice were phenotypically indistinguishable from their WT littermates in terms of gross morphology, whereas homozygous $\operatorname{Lrc} 8 a^{\mathrm{F} 443^{*} / \mathrm{F} 443^{*}}$ mice exhibited curly vibrissae 
A

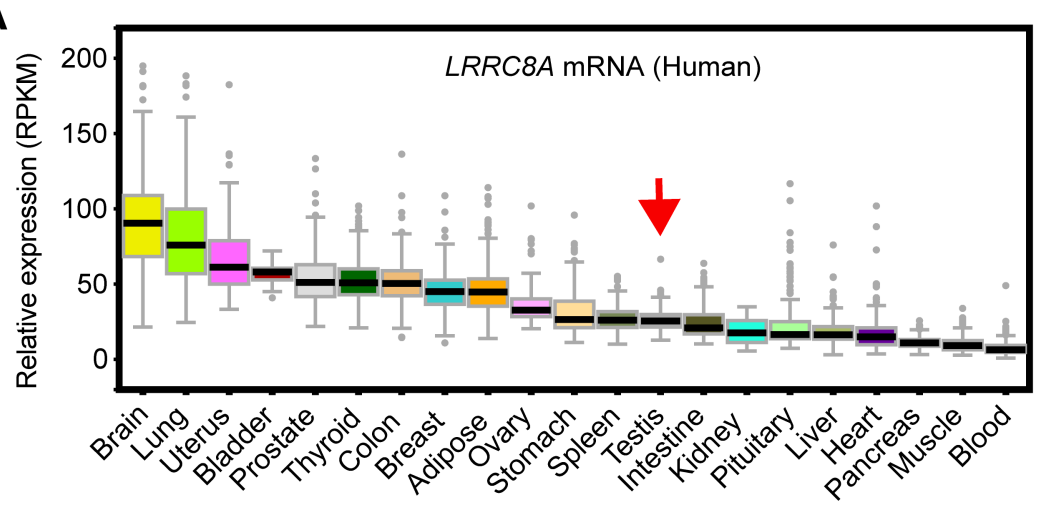

B

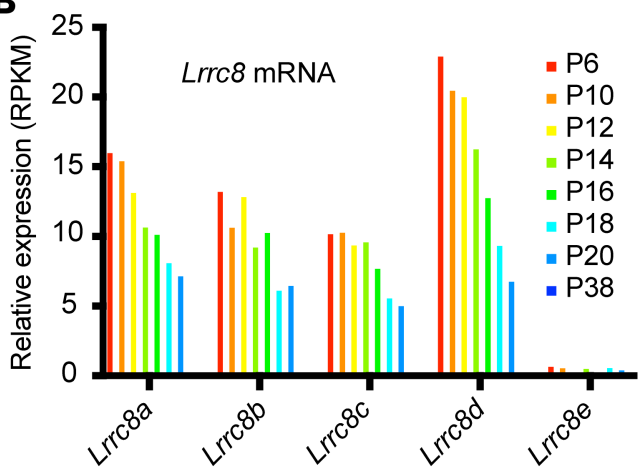

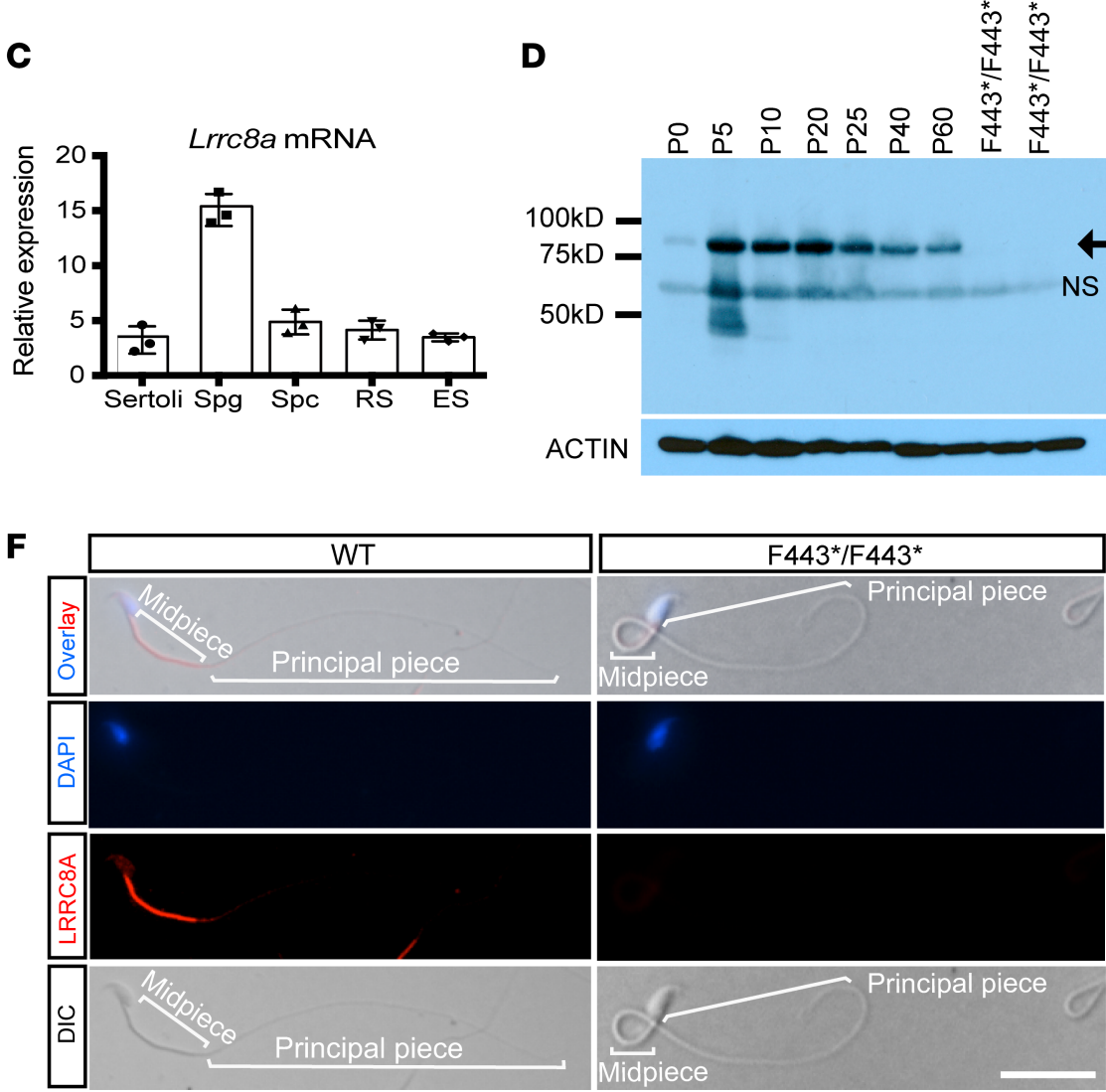

E
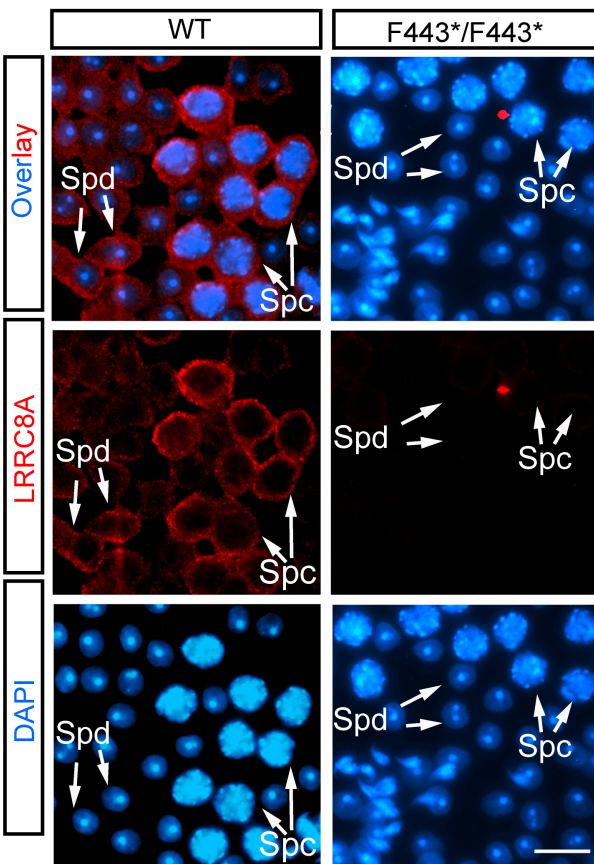

Figure 1. Expression and localization of LRRC8A. (A) Relative median expression levels of $L R R C 8 A$ mRNA in different human tissues as detected by RNA-seq according to the GTEx database. Tissues were collected from 570 adult postmortem individual donors. RPKM, reads per kilobase of transcript per million. (B) Relative mRNA expression levels of Lrrc8 paralogs (Lrrc $8 a-e$ ) in the developing mouse testis during postnatal development (calculated from Gene Expression Omnibus [GEO] accession number GSE44346). (C) qPCR analysis of Lrrc8a mRNA levels among the purified Sertoli cells and different subtypes of germ cells inside the seminiferous tubules from 3 biological replicates. Spg, spermatogonia; Spc, spermatocyte; RS, round spermatid; ES, elongating spermatid. Data were presented as mean \pm SD $(n=3)$. (D) LRRC8A protein expression during postnatal testicular development in mice. Protein lysates from homozygous $L r r c 8 a^{F 443^{*} / F 443^{*}}$ mice testes were used as negative controls. The arrow points to the LRRC8A-specific protein band of expected size, which is absent in the Lrrc $8 a^{\mathrm{F} 443^{*} / F 443^{*}}$ lanes. NS, nonspecific band. ACTIN serves as an internal control. (E) Representative immunofluorescence (IF) images of LRRC8A in germ cell spreads of the seminiferous tubules by squash preparation. LRRC8A protein is present in the plasma membrane of Spc and spermatids (Spd). Testis from F443*/F443* mice were used as negative controls. Scale bar: $20 \mu \mathrm{m}$. (F) LRRC8A protein localization by immunofluorescence in mature sperm from WT and F443*/F443* cauda epididymis. LRRC8A is intensively labeled in the midpiece, with weak labeling in the head and principal piece of mature sperm. Sperm from F443*/F443* cauda served as negative controls. Scale bar: $5 \mu \mathrm{m}$. DIC, differential interference contrast. 
and a characteristic wavy, sparse coat starting at around P7 (data not shown) and tended to die by approximately 3 months of age (29). When intercrossed with fertility-proven WT females, male $L r r c 8 a^{\mathrm{F} 443^{*} / \mathrm{F} 443^{*}}$ mice produced no pups over a 6-month breeding period for the survivors. The testis weight of $\operatorname{Lrc} 8 a^{\mathrm{F} 443^{*} / \mathrm{F} 443^{*}}$ mice was slightly lower than that of WT littermates at 6 weeks of age (Figure 2, A-C), but the sizes of seminal vesicles were indistinguishable, suggesting that testosterone secretion by the Leydig cells was not impacted in Lrrc8 $a^{\mathrm{F} 443^{*} / \mathrm{F} 43^{*}}$ males (Figure 2C). Notably, there were severe morphological defects in the flagella of mature sperm retrieved from the cauda epididymis in the $\operatorname{Lrc} 8 a^{\mathrm{F} 443^{*} / \mathrm{F} 443^{*}}$ males, such as headless/acephalic sperm, short tail, curly midpiece, and intertwined tails around the neck, as partially described in our previous study (28). These features are reminiscent of the MMAF phenotype observed in clinically infertile human patients (Figure 2D) (37). Overall, these irregularly shaped sperm mostly displayed curly midpieces and tails, which contrasted sharply with the typical hook-type sperm observed in WT controls (Figure 2, D and E). Both scanning electron microscopy and transmission electron microscopy (TEM) further confirmed the MMAF anomalies that account for $\operatorname{Lrc} 8 a^{\mathrm{F} 443^{*} / \mathrm{F} 43^{*}}$ male infertility (Figure 2, F and G). Notably, the major defect causing the MMAF-like phenotype is likely due to the disruption of volume regulation in the $\operatorname{Lrc} 8 a^{\mathrm{F} 443^{*} / \mathrm{F}_{4} 3^{*}}$ mutants, causing a wide range of previously described secondary effects (28), which we investigated next.

Given that Lrrc8a is expressed in both Sertoli cells and all germ cell subtypes in testis, we investigated the functional consequences of the $\operatorname{Lrc} 8 a^{\mathrm{F} 443^{*}}$ mutant allele on germ cell development. H\&E staining revealed that, while the numbers of different types of germ cells (e.g., pachytene spermatocytes, round spermatids, and elongating spermatids) were comparable between the WT and $\operatorname{Lrc} 8 a^{\mathrm{F} 443^{*} / \mathrm{F} 443^{*}}$ testis at 6 weeks of age (Figure 3A), a large quantity of vacuoles was observed in $\operatorname{Lrrc}^{8 a^{\mathrm{F} 443^{*} / \mathrm{F} 443^{*}}}$ testis, presumably due to the sloughing of affected germ cells at 3 months of age (Figure 3A). A detailed histological examination demonstrated that the affected cells encompass spermatogonia, spermatocytes, and spermatids (Figure 3A). These results suggest that $\operatorname{Lrc} 8 a$ is required for the survival of different types of germ cells.

The deformed sperm tail morphology observed in $\operatorname{Lrc} 8 a^{\mathrm{F} 443^{*} / \mathrm{F} 43^{*}}$ cauda epididymis was largely attributed to the defective haploid spermatid development, when the sperm flagella forms in the seminiferous tubules. Thus, we carried out TEM imaging of spermatids from testis tissue to examine morphological alterations in $\operatorname{Lrc} 8 a^{\mathrm{F} 443^{*} / \mathrm{F} 43^{*}}$ males. Remarkably, and consistent with the role of LRRC8A in volume regulation, the cytoplasm of round spermatids from $\operatorname{Lrc} 8 a^{\mathrm{F} 443^{*} / \mathrm{F} 443^{*}}$ mutants is swollen compared with that of WT, as determined by the nuclear-to-cytoplasmic volume ratio (NC ratio) (Figure 3, B and C). Likewise, the shedding cytoplasmic volume in the elongating spermatids is greater in $\operatorname{Lrc} 8 a^{\mathrm{F} 443^{*} / \mathrm{F} 43^{*}}$ males than in WT. This is unlikely attributed to the compromised phagocytosis of Sertoli cells, since this phenomenon was also observed in germ cell-specific Lrrc8a-cKO testis (Figure 3, B and C). Moreover, a fraction of spermatids displayed curly midpieces and tails, and even disconnected tails, as observed in cauda epididymis (Figure 3, B and C). Together, these data demonstrate that Lrrc $a$ is essential for germ cell development in the testis, at least in part, through its volume-regulating capability.

Diminished VRAC activity in the Lrrc $8 a^{F 443^{*} / F 443^{*}}$ germline. Dysfunctional volume regulation within germ cells inside the testis of $L r r c 8 a^{\mathrm{F} 443^{*} / \mathrm{F} 443^{*}}$ mutants implies that VRAC activity is impaired in these germ cells. To test this hypothesis, we performed whole-cell recordings on acutely dissociated, primary germ cells isolated from testis. We chose spermatocytes as a representative cell type, since their large size distinguishes them from other cell types. When subjected to hypotonic stress $(\sim 220 \mathrm{mOsm} / \mathrm{kg})$, we observed a timedependent increase in VRAC current at $+80 \mathrm{mV}$, and a decrease at $-80 \mathrm{mV}$. In contrast, hypotonic stressinduced VRAC currents were diminished in spermatocytes from $\operatorname{Lrc} 8 a^{\mathrm{F} 443^{*} / \mathrm{F} 443^{*}}$ mice (Figure $4, \mathrm{~A}$ and B).

Next, we measured VRAC currents in spermatocytes elicited by a set of depolarizing potentials ranging from $-70 \mathrm{mV}$ to $+140 \mathrm{mV}$, with $10 \mathrm{mV}$ intervals starting from a holding potential of $-70 \mathrm{mV}$. Voltage steps were recorded 5 minutes after bath application of hypotonic solution. The amplitude of whole-cell outward currents showed a voltage-dependent increase, and the current density at $+140 \mathrm{mV}$ was large in spermatocytes from WT mice (Figure 4, C and D). In contrast, the current density was markedly reduced in spermatocytes from $\operatorname{Lrc} 8 a^{\mathrm{F} 443^{*} / \mathrm{F} 443^{*}}$ mice (Figure 4, C and D).

To define the ionic nature of the current, we measured the reversal potential during the peak activation of the current by determining current-voltage (I-V) relationships. To eliminate the confounding contribution of voltage-gated cationic currents, we used N-methyl-D-glucamine (NMDG) to replace all permeable cations. When the cells were maintained in a permeable cation-free solution, the I-V curves during the peak response to low osmolality had a reversal potential at 0 at equimolar intra- and extracellular $\mathrm{Cl}^{-}(100 \mathrm{mM})$, with a small degree of outward rectification (Figure 4, E and F). Reducing extracellular $\mathrm{Cl}^{-}$from $100-50 \mathrm{mM}$, and then to 
A

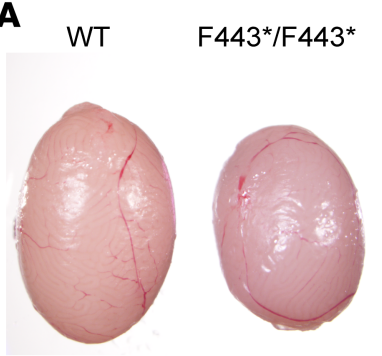

B

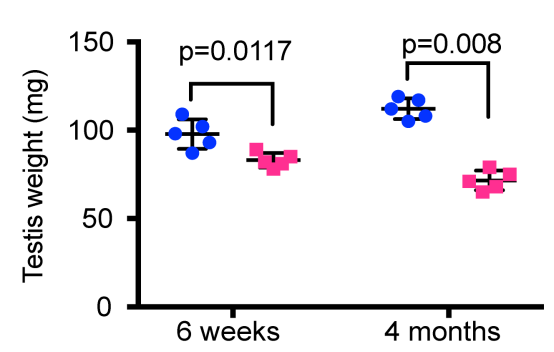

- WT

F443*/F443*
C

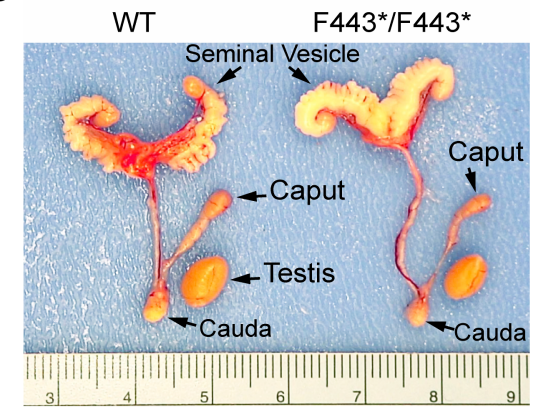

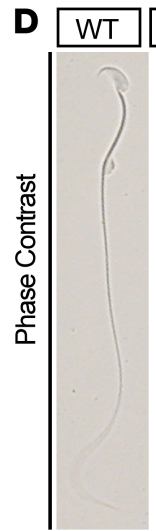

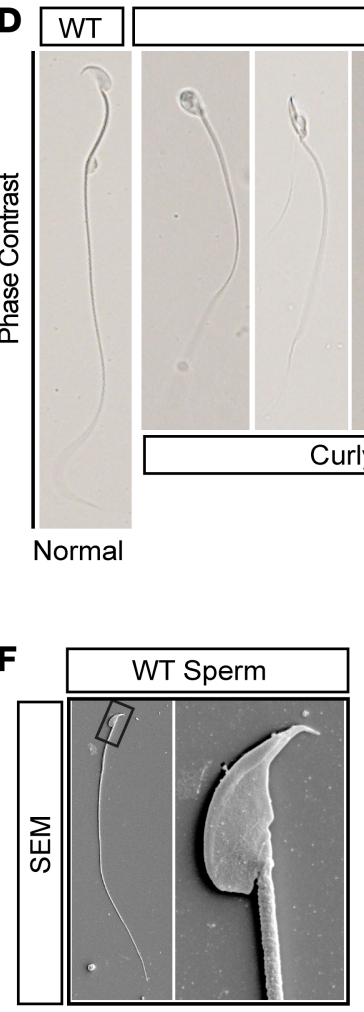

$\mathbf{F}$

G

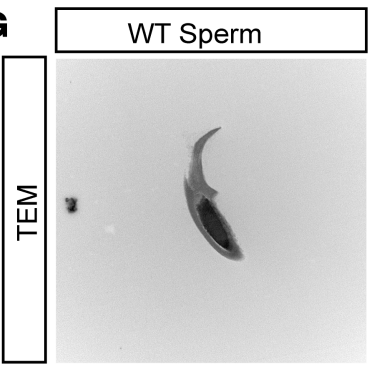

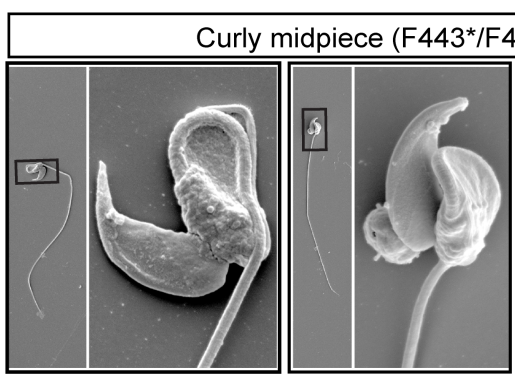
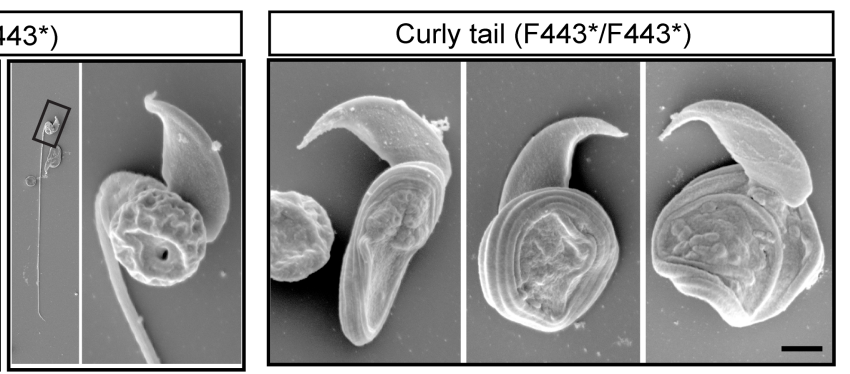

E

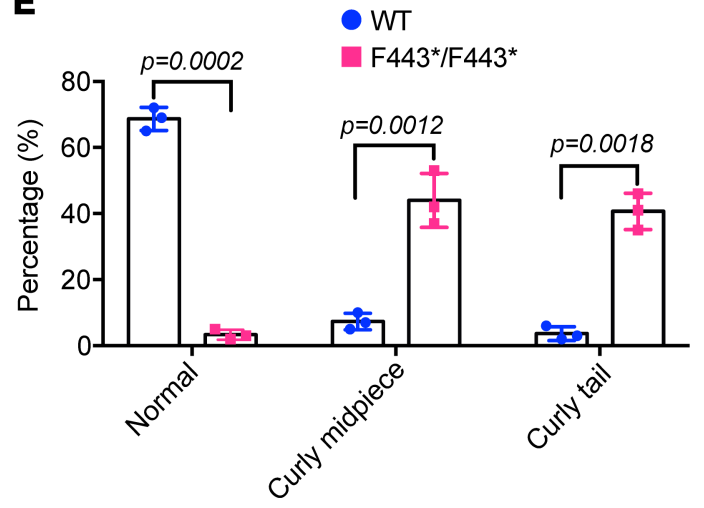

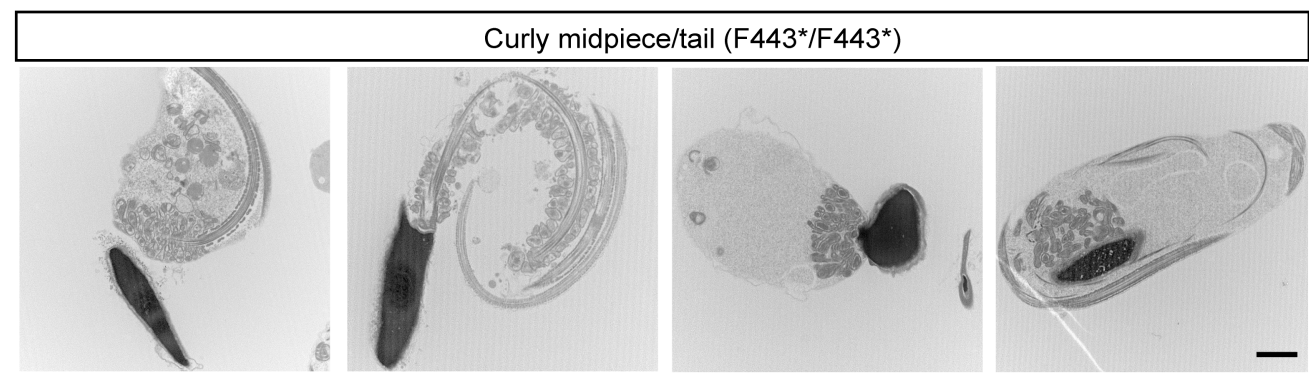

Figure 2. Phenotypic characterization of $L r r c 8 a^{\mathrm{F}^{443^{*} / \mathrm{F}_{443}}}$ male reproductive tract and sperm. (A) Gross morphology of testis from WT and $L r r c 8 a^{\mathrm{F} 443^{*} / \mathrm{F} 443^{*}}$ mice at 6 weeks of age. (B) Comparison of the testis weight between WT and $L r r C 8 a^{\text {F } 443^{*} / F 443^{*}}$ mice at 6 weeks and 4 months of age. Data represent the

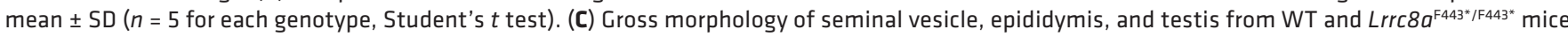
at 8 weeks of age. (D and $\mathbf{E}$ ) Morphology of sperm retrieved from the cauda epididymis in WT and $L r r c 8 a^{F 443^{*} / F 443^{*}}$ mice. WT sperm typically harbor a "hook" head and straight tail. Greater than $95 \%$ of sperm from Lrrc8 $a^{\mathrm{F} 443^{*} / \mathrm{F}_{4} 3^{*}}$ cauda display an aberrant morphology, primarily curly midpieces and tails (E) ( $n=5$ for each genotype, Student's $t$ test). Scale bar: $3 \mu \mathrm{m}$. (F) Representative scanning electron microscopy images showing morphology of

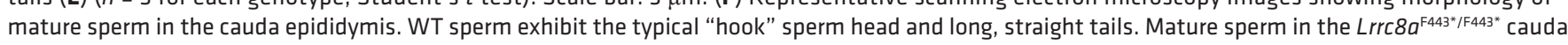
display mostly twisted, curly midpieces or curly tails. Scale bar: $1 \mu \mathrm{m}$. (G) Representative TEM images showing the morphology of mature sperm in the cauda epididymis. Mature sperm in the $L r r c 8 a^{F 443^{*} / F 443^{*}}$ cauda display mostly retroflexed, curly midpieces or curly tails. Note that there is no cytoplasmic mass wrapped around the sperm neck in WT. Scale bar: $1 \mu \mathrm{m}$. 
A

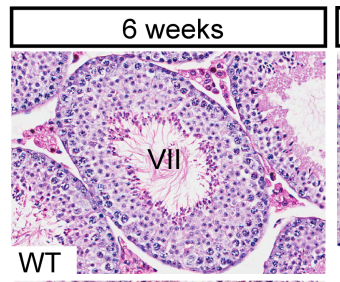

WT

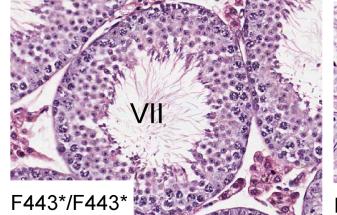

$F 443^{+} F 443^{*}$ the

N.6. S.
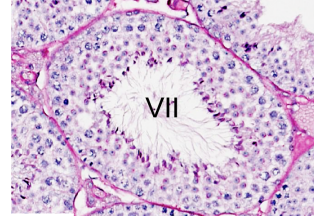

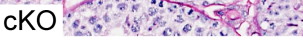
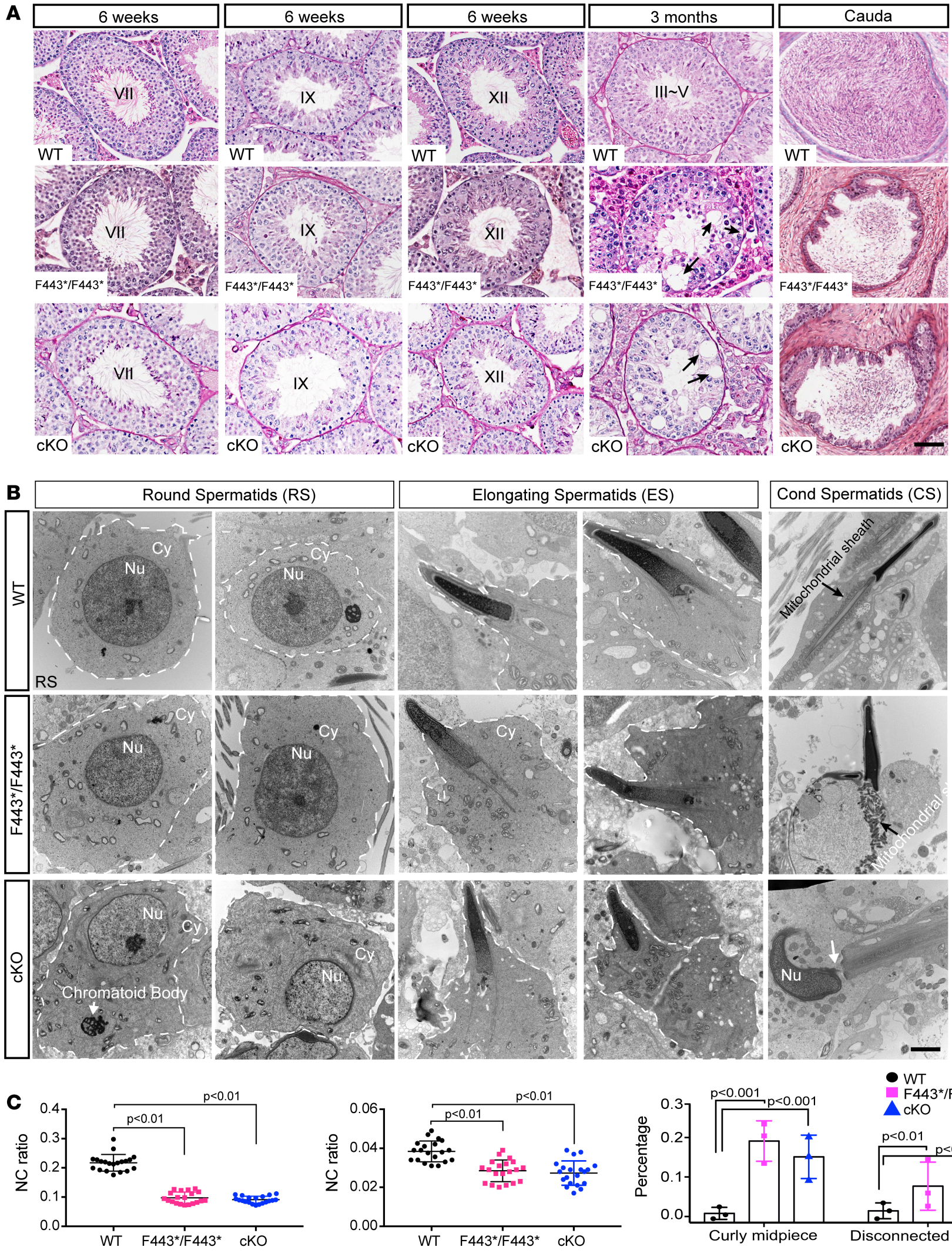

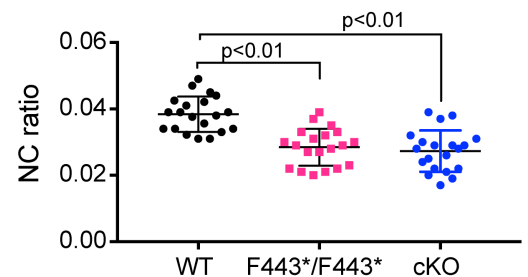

Elongating Spermatids (ES)

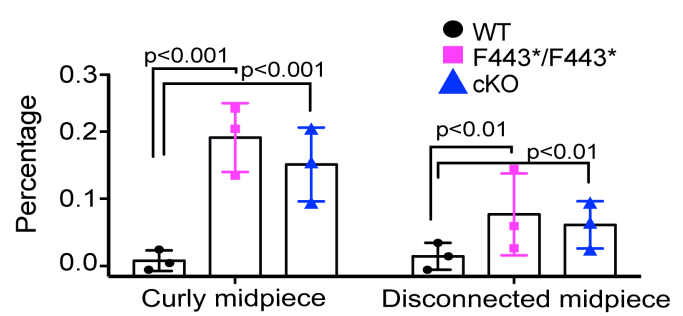

Condensed Spermatids (CS)

Figure 3. Histopathology of testis in the $\operatorname{Lrrc8}^{\mathrm{F} 443^{*} / F 443^{*}}$ mice. (A) H\&E staining of the testicular cross-sections in WT, Lrrc $8 a^{\mathrm{F} 443^{*} / F 443^{*}}$, and germ cell-specific Lrrc8a KO (cKO) mice at different time points. Representative stages (indicated in Roman numerals) are shown. Images in the fifth column show the H\&E staining of the cauda epididymis from mice at 8 weeks of age. Arrows point to the vacuoles observed inside the tubules. Scale bar: $5 \mathrm{~mm}$. (B) Representative TEM images showing the morphology of germ cells inside the testis. Enlarged cytoplasm, disconnected sperm 
heads (white arrow), and curly tails were observed in sperm from Lrrc $8 a^{\mathrm{F} 443^{*} / \mathrm{F} 443^{*}}$ and Lrrc $8 a$-cKO testis. RS, round spermatids; ES, elongating spermatids; CS, condensed spermatids; Nu, nucleus; Cy, cytoplasm. The dashed line delineates the nuclear membrane. Scale bar: 10 mm. (C) Statistical comparisons of the nuclear-to-cytoplasmic volume ratio (NC) for round spermatids (left panel) and elongating spermatids (middle panel) from 3 male testes among different cohorts of genotypes as indicated (ANOVA, $n=21$ for total number of germ cells calculated in each genotype). The NC ratio represents the ratio of NC volume as estimated from 2-dimensional cross-section TEM images using the formula described in methods. The percentages of deformed morphology of condensed spermatids (either curly midpiece or disconnected midpiece) were calculated from 3 biological testis samples as visualized by TEM among 3 genotypes (ANOVA, $n=27$ for total number of spermatids calculated in each genotype) (right panel). Data represent the mean \pm SD.

$4 \mathrm{mM}$, shifted the reversal potential progressively to a more positive voltage in spermatocytes from WT mice (Figure 4, E and F). In contrast, lowering the extracellular $\mathrm{Cl}^{-}$concentration did not shift the reversal potential of currents in spermatocytes from $\operatorname{Lrc} 8 a^{\mathrm{F} 443^{*} / \mathrm{F} 443^{*}}$ testis (Figure 4, E and F). Taken together, these results indicate that $\operatorname{Lrc} 8 a^{\mathrm{F} 443^{*} / \mathrm{F} 443^{*}}$ mutation diminishes LRRC8A-dependent VRAC activity of germ cells in the testis.

The Lrrc $8 a^{F 443^{*}}$ mutation does not affect oogenesis, but embryos derived from Lrrc $8 a^{F 443^{*} / F 443^{*}}$ females show early developmental failure. In addition to causing male infertility, we observed that $\operatorname{Lrc} 8 a^{\mathrm{F} 443^{*} / \mathrm{F} 443^{*}}$ females also failed to produce pups when intercrossed with fertility-proven WT males. Therefore, we next probed whether the $\operatorname{Lrrc} 8 a^{\mathrm{F} 443^{*}}$ mutation affected oogenesis. H\&E staining of paraffin-embedded ovary sections from 2-month-old Lrrc8 $a^{\mathrm{F} 443^{*} / \mathrm{F} 443^{*}}$ and WT mice revealed comparable numbers of follicles at different stages with normal morphology (data not shown). Fully grown germinal vesicle (GV) oocytes, isolated from the ovaries of pregnant mare serum gonadotropin-primed (PMSG-primed) mice, and mature metaphase II (MII) oocytes, retrieved from the oviducts of superovulated mice, also showed no differences in morphology or number between the Lrrc $8 a^{\mathrm{F} 443^{*} / \mathrm{F} 43^{*}}$ and WT females (Supplemental Figure 2A). To investigate whether there is a defect in meiotic resumption and progression, we isolated GV oocytes from PMSG-primed ovaries and performed in vitro meiotic maturation assays. After 24 hours of culture, both genotypes had comparable percentages of oocytes at the GV, MI, and MII stages between the 2 genotypes, albeit with a slightly, but not significantly, larger number of fragmented oocytes from $\mathrm{F} 443^{*} / \mathrm{F} 443^{*}$ females (Supplemental Figure 2B). These data strongly suggest that Lrrc8a deficiency has no major impact on oogenesis, per se.

To study whether the infertility of $\operatorname{Lrrc} 8 a^{\mathrm{F} 443^{*} / \mathrm{F} 443^{*}}$ females is due to defects in embryogenesis, we monitored preimplantation embryonic development in vitro. Superovulated $\operatorname{Lrc} 8 a^{\mathrm{F} 443^{\star} / \mathrm{F} 443^{*}}$ and WT females were mated with WT males, and zygotes collected at E0.5 were cultured for 24-72 hours. While oocytes from $\operatorname{Lrc} 8 a^{\mathrm{F} 443^{*} / \mathrm{F} 443^{*}}$ females could be fertilized by WT sperm, the number of zygotes recovered was significantly reduced compared with WT controls (Supplemental Figure 2, C and D). Detailed examination at multiple time points demonstrated that, while the vast majority ( $>90 \%$ ) of fertilized WT oocytes proceeded through the 2-cell, 4-cell, 8-cell, and morula stages and ultimately reached the blastocyst stage, fertilized oocytes from $\operatorname{Lrc} 8 a^{\mathrm{F} 443^{*} / \mathrm{F} 443^{*}}$ females exhibited severe developmental delays and progressive fragmentation/ degeneration, with none developing beyond the 8-cell stage (Supplemental Figure 2E). This resulted in the complete absence of embryo implantation sites in $\operatorname{Lrc} 8 a^{\mathrm{F} 443^{*} / \mathrm{F} 443^{*}}$ females after natural mating, in sharp contrast to the numerous sites observed in WT females, as determined by the blue dye staining in the uterus (Supplemental Figure 2F). Therefore, early developmental failure accounts for the infertility phenotype of $\operatorname{Lrrc} 8 a^{\mathrm{F} 443^{*} / \mathrm{F} 443^{*}}$ female mice. Thus, we conclude that $\operatorname{Lrrc} 8 a$ is dispensable for oogenesis but is required by the oocytes for embryonic development upon fertilization.

Constitutive and germline-specific Lrrc8a-KO mouse models recapitulate the phenotypic defect of the germ cells in Lrrc $8 a^{F 443^{*} / F 443^{*}}$ males. Despite the dramatically reduced Lrrc8a mRNA levels in $L r r c 8 a^{\mathrm{F} 443^{*} / \mathrm{F} 443^{*}}$ testis, it is possible that a truncated LRRC8A protein is present in the mutant testis. Therefore, we generated 2 new genetically engineered mouse models (GEMMs), a constitutive Lrrc8a-KO mouse and a germline-specific Lrrc $8 a$-cKO, to fully uncover the role of $L r r c 8 a$ in testicular development. The constitutive $L r r c 8 a-K O$ mice has the exons 3 and 4 replaced by a Neo cassette bearing an upstream LacZ reporter (Figure 5A). Using LacZ reporter as a surrogate for $\operatorname{Lrc} 8$ a expression, we found that LRRC8A is expressed in all cell types in testis, consistent with our qPCR and immunolabeling results (data not shown). Homozygous Lrrc $8 a-$ KO pups exhibited a higher incidence of perinatal mortality $(\sim 22 \%)$, compared with $L r r c 8 a^{\mathrm{F} 443^{*} / \mathrm{F}_{4} 43^{*}}$ pups $(\sim 6 \%)$, but both had similar defects in spermatogenesis in testis (data not shown) and MMAF-like sperm morphology in cauda epididymis (Figure $5 \mathrm{C}$ ).

As mentioned previously, Lrrc8a is ubiquitously expressed in all cell types in testis, and thus, the infertility phenotype might not be germline-specific but might result from a combination of defects in Lrrc 8 a function in multiple cell types. To exclude the somatic contribution of LRRC8A to the defective spermatogenesis in the 

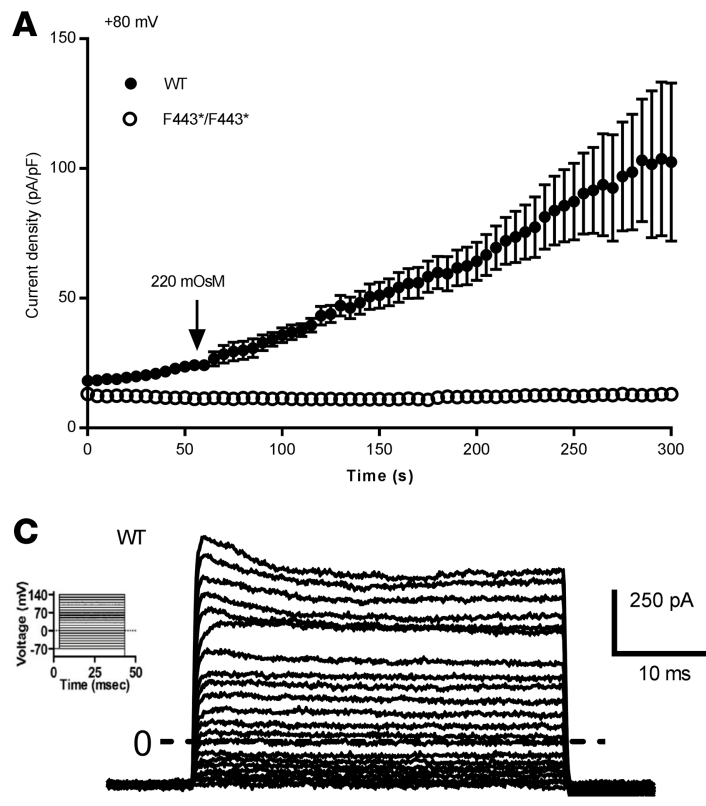

$\mathrm{F} 443^{*} / \mathrm{F} 443^{*}$

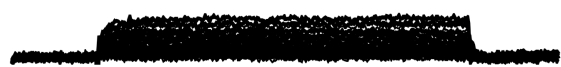

$\mathbf{E}$

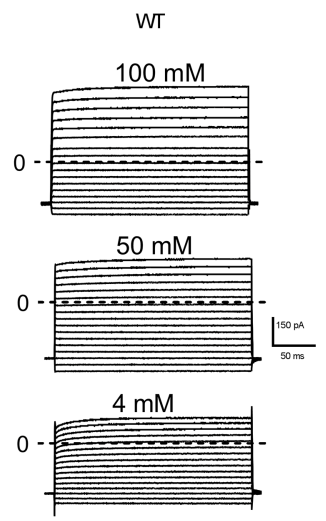

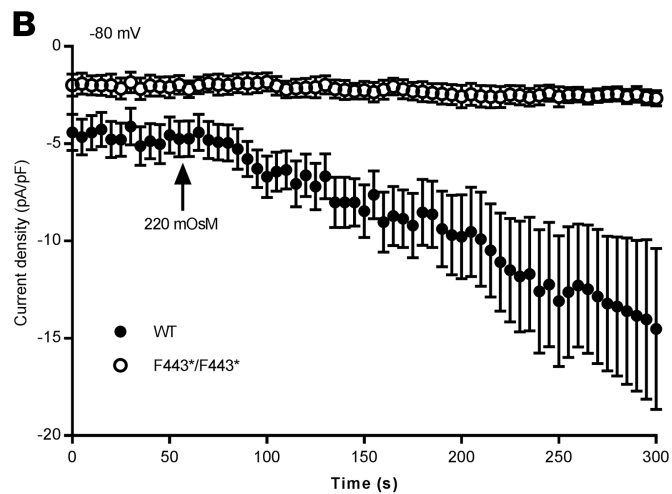

D

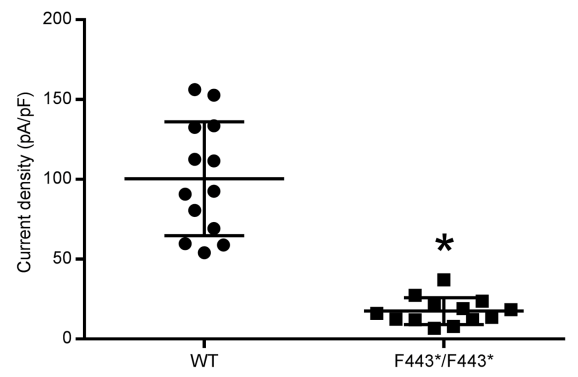

$\mathbf{F}$

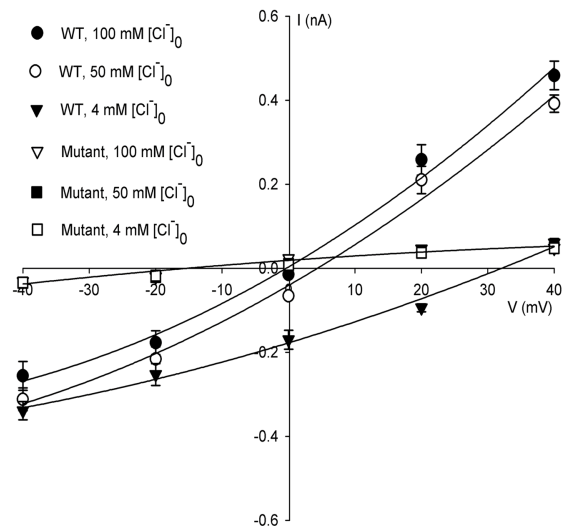

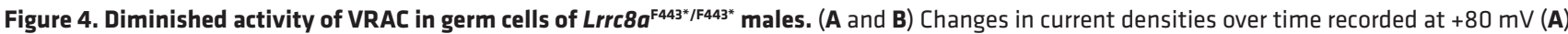
or $-80 \mathrm{mV}(\mathbf{B})$ in spermatocytes obtained from WT controls and Lrrc8 $a^{\mathrm{F} 443^{*} / F 443^{*}}$ mice after switching to a hypotonic solution ( $n=9$ in each group). Data represent mean \pm SEM. (C) Representative current traces in spermatocytes from WT (upper panel) and $L$ rrc8 $a^{\mathrm{F} 443^{*} / \mathrm{F} 443^{*}}$ testis (lower panel). Inset, voltage step protocol. (D) Quantification of current densities elicited at $+140 \mathrm{mV}$ in spermatocytes from WT and $L r r c 8 a^{\mathrm{F} 443^{*} / \mathrm{F} 443^{*}}$ mice ( $n=13$ in each group). Data are shown as mean \pm SEM. ${ }^{*} P<0.001$ (Student's $t$ test). (E) Original current traces recorded in different extracellular $\mathrm{Cl}^{-}$concentrations in spermatocytes

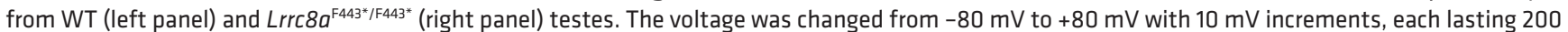
ms with an interval of 1 second. The holding potential was $-70 \mathrm{mV}$. Dashed lines indicate zero current. (F) Current-voltage (I-V) relationships of maximally activated currents. The I-V relationship shows a reversal potential near 0 at equimolar intra- and extracellular $\mathrm{Cl}^{-}$of 100 mM. There is a progressive shift to more positive potentials with reduced extracellular $\mathrm{Cl}^{-}$from $100 \mathrm{mM}$ to $50 \mathrm{mM}$ and then to $4 \mathrm{mM}$ in spermatocytes from WT testes ( $n=10$ ). This shift is

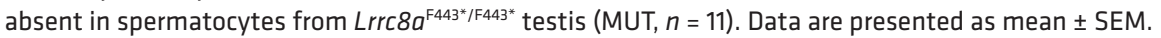

Lrrc8a-KO mice, we generated a germline-specific Lrrc8a-cKO, in which Stra8-driven Cre excises a floxed exon 3 exclusively in germ cells of testis from P3 onward (Figure 5B). Indeed, we found that sperm retrieved from the cauda epididymis of all 3 mouse models - F443*/F443*, KO, and cKO - exhibited similar MMAF-like phenotypes (Figure 5C), and their spermatids had a lower NC ratio in the testis (Figure 3C). These data validated a germ cell-autonomous role for $\operatorname{Lrc} 8 \mathrm{a}$ that is essential for spermatogenesis and male fertility.

Identification of a hypomorphic LRRC8A mutation (c.1634G>A, p. Arg545His) in a sterile male patient. Recent efforts with high-throughput whole-exome sequencing (WES) have identified genetic mutations (e.g., missense mutations, nonsense mutations, deletions, or insertions) in dozens of genes that are closely linked to idiopathic human infertility (38). To interrogate a possible link between LRRC $8 A$ mutations 
A

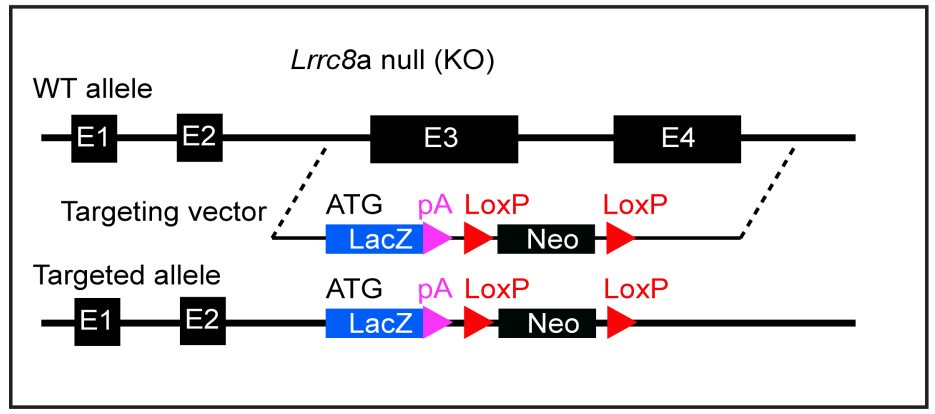

C
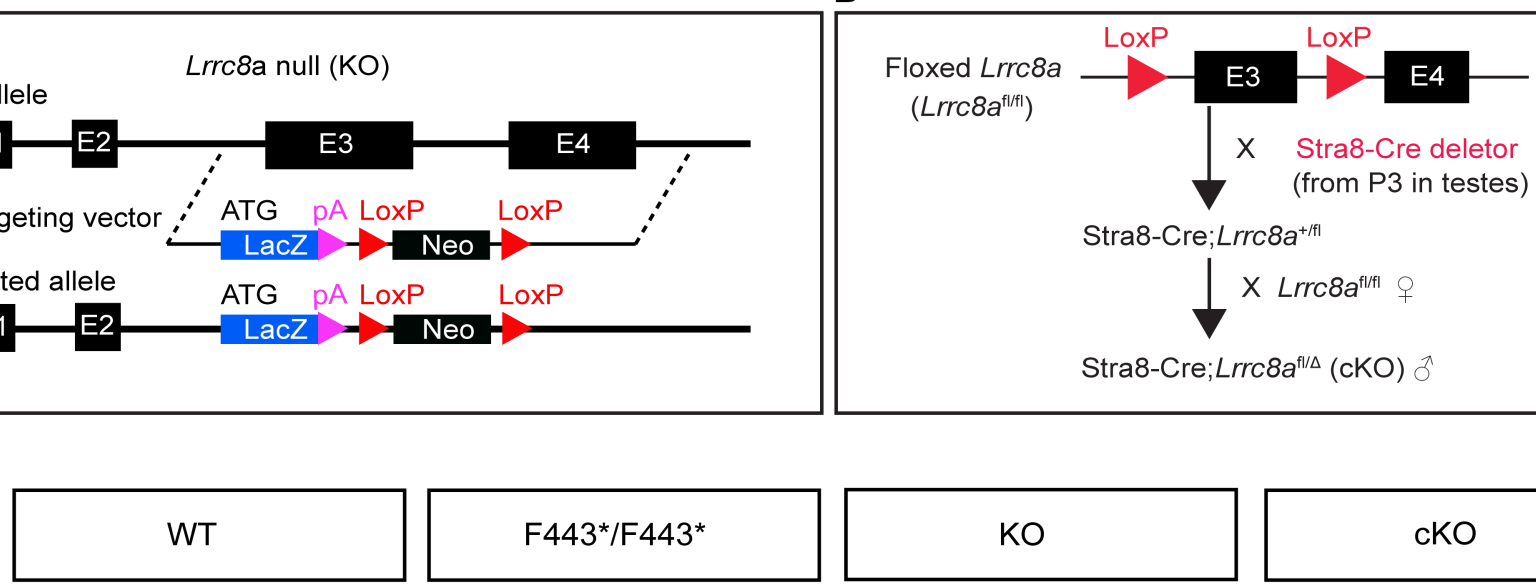

Stra8-Cre; Lrrc8a $a^{+/ f 1}$

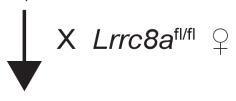

Stra8-Cre; Lrrc8a ${ }^{\text {f/L }}(\mathrm{cKO})$
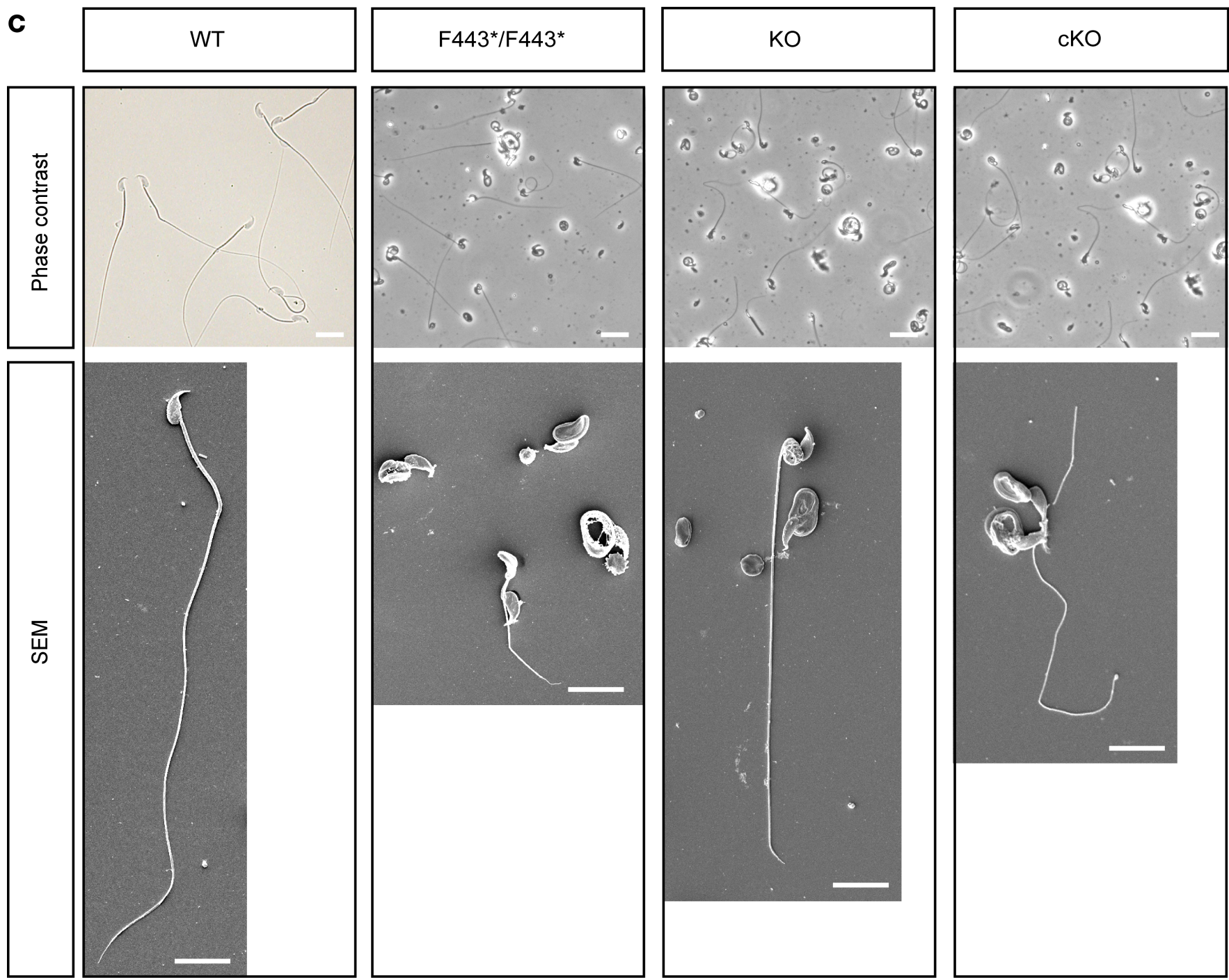

Figure 5. Constitutive and germline-specific null mutations of Lrrc8a cause deformed sperm morphology (multiple morphological abnormalities of the flagella; MMAF) similar to that of $\operatorname{Lrc8} 8 a^{\mathrm{F443} / F 443^{*}}$ mice. (A) Schematic diagram showing the construct design for making Lrrc $8 a$-null alleles

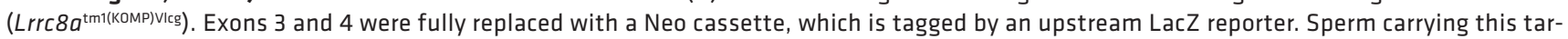
geted allele were used to fertilize WT oocytes in vitro. Fertilized eggs were transferred to pseudo-pregnant females to produce constitutive Lrrc8a$\mathrm{KO}$ mice (designated as KO). The insertion of the LacZ/Neo cassette abolished Lrrc8a gene expression. (B) Sperm harboring the targeted conditional

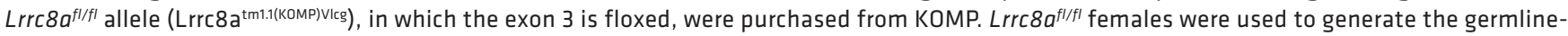
specific Lrrc8a KO (cKO) mice, by breeding with a germline-specific Cre line (Stra8-Cre) (designated as Stra8-Cre; Lrrc8a f// [cKO]). (C) Representative morphology of sperm visualized by phase contrast (top; scale bar: $3 \mu \mathrm{m}$ ) and scanning electron microscopy (bottom; scale bar: $2 \mu \mathrm{m}$ ), retrieved from the cauda epididymis in the WT, $\operatorname{LrC} 8 a^{\mathrm{F} 443^{*} / F 443^{*}}$, KO, and cKO mice. 
and human infertility, we performed WES on a cohort of 102 patients diagnosed with Sertoli cell-only syndrome (SCOS), a disease manifested by an absence of germ cells leaving only Sertoli cells in the testis. We chose SCOS patients because the LRRC8A-deficient germ cells in our mouse models degenerate during postnatal development, leading to a SCOS-like phenotype. Genomic DNA from the peripheral blood of SCOS patients was extracted and sequenced, and clean reads were aligned to the human reference genome (hg19). To identify putative causative mutations, variants meeting the following criteria were subjected to further analyses: (a) nonsense, missense, frameshift, or splicing variants; (b) absent or rare in dbSNP (http://www.ncbi.nlm.nih.gov/snp/), the 1000 Genomes (http://www.internationalgenome. org/), and the ExAC databases (http://exac.broadinstitute.org/) (all with minor allele frequency [MAF] $<0.05$ ); and (c) absent in our in-house WES database (254 healthy, fertile, Han Chinese men and 283 healthy, fertile, European-descent men). As a result, we identified only a single heterozygous $L R R C 8 A$ missense variant (NM_001127244.1, c.1634G>A, p. Arg545His) in a single patient (patient 90) (Figure 6A), which was verified by Sanger sequencing (Figure 6B). No other variants meeting our criteria were detected in this patient. In sharp contrast to normal male controls, H\&E staining of a testicular biopsy from this patient showed an absence of germ cells and the presence of atypical Sertoli cells that aggregated inside the seminiferous tubules (Figure 6C).

The LRRC8A variant (c.1634G>A) (dbSNP: rs769167142) is a rare allelic mutation with no record in either the 1000 Genome project database or in our in-house WES database of healthy, fertile men, but it is present with a frequency of 0.000008340 in the ExAC database. Its rarity suggests that it is highly likely a deleterious, causative mutation. Indeed, the incidence of this mutant allele in our SCOS cohort was 1/204 (i.e., 0.0049), much higher than the MAF recorded in ExAC, likely reflecting the distinct human populations (Asian population vs. European population) in these datasets. The $\mathrm{G}$ to A substitution led to the conversion of a highly conserved Arginine $(\mathrm{R})$ to Histidine $(\mathrm{H})$ at amino acid $545\left(L R R C 8 A^{\mathrm{R} 545 \mathrm{H}}\right)$, located within the seventh LRR domain (Figure 6A). In silico analyses using multiple computational programs, such as PolyPhen (http://genetics.bwh.harvard.edu/pph2/), MutationTaster, SiPhy, and LRT, predicted that the $L R R C 8 A^{\mathrm{R} 545 \mathrm{H}}$ mutation is deleterious (39). We further carried out 3-dimensional homology modeling of LRRC8A protein structure based on the sequence homology with the closest LRR domain homologs in the Protein Data Bank (PDB) (40). As shown in Figure 6D, the $L R R C 8 A^{\mathrm{R} 545 \mathrm{H}}$ mutation substantially impacted both the topological structure of the transmembrane domains and the distribution of the LRR domains, which could potentially abrogate chloride channel activity or downstream signaling.

To pinpoint the effect of this mutation on VRAC activity, we performed in vitro current recordings by utilizing our previously established Xenopus oocyte expression system (41). In this system, we have previously validated that simultaneous expression of fluorescently tagged LRRC8A, along with other LRRC8 subunits (except for LRRC8B), generates persistent currents under isosmotic conditions, which are further reinforced under hypotonic conditions (41). Since LRRC8E is not detectable in testis (Figure 1), we thus tested the combinations for LRRC8A/C and LRRC8A/D. Human LRRC8A containing a C-terminal VFP tag (8A-VFP) and either LRRC8C or LRRC8D fused at the C-terminus with mCherry ( $\mathrm{mCh}$ ) (abbreviated as $8 \mathrm{C}-\mathrm{mCh}$ and $8 \mathrm{D}-\mathrm{mCh}$, respectively), were coexpressed in Xenopus oocytes through injection of their respective cRNAs. In comparison with WT 8A-VFP expression, mutant $8 \mathrm{~A}(\mathrm{R} 545 \mathrm{H})$-VFP induced a significant decrease in the intensity of the current when coinjected with $8 \mathrm{C}-\mathrm{mCh}$ or $8 \mathrm{D}$-mCh, as evidenced by the typical current traces (Figure 6E). Remarkably, 8A(R545H)-VFP injection evoked a prominent reduction of the current intensity by approximately $25 \%-30 \%$ as compared with the WT $8 \mathrm{~A}-\mathrm{VFP}$, when coinjected with either $8 \mathrm{C}-\mathrm{mCh}$ or $8 \mathrm{D}$-mCh, independently (Figure $6 \mathrm{~F}$, left). The current reduction in the LRRC8A $(\mathrm{R} 545 \mathrm{H})$ mutant was not due to changes in protein expression levels because Western blot validated that the cRNAs for WT 8A-VFP and mutant 8A(R545H)-VFP produced comparable levels of proteins (Figure $6 \mathrm{~F}$, right). These data suggest that $L R R C 8 A^{\mathrm{R} 545 \mathrm{H}}$ is a hypomorphic mutation. To further substantiate these findings, we coinjected tandemly tagged 8A (HA-VFP tag) with either $8 \mathrm{C}-\mathrm{mCh}$ or $8 \mathrm{D}$-mCh, and we consistently found a reduction in current of about $30 \%$ in $L R R C 8 A^{\mathrm{R} 545 \mathrm{H}}$ compared with in WT LRRC8A (Figure 6G). This reduction was solely ascribed to change of VRAC activity in $L R R C 8 A^{\mathrm{R} 545 \mathrm{H}}$, since WT and mutant LRRC8A proteins were expressed at similar levels (Figure 6G, right). Next, to determine whether $L R R C 8 A^{\mathrm{R} 545 \mathrm{H}}$ acts as a dominant-negative mutation, we coinjected a 1:1 mixture of tagged WT (50\%) and $L R R C 8 A^{\mathrm{R} 545 \mathrm{H}}(50 \%)$, along with either $8 \mathrm{C}-\mathrm{mCh}$ or $8 \mathrm{D}-\mathrm{mCh}$. In this system, LRRC8 $\mathrm{A}^{\mathrm{R} 545 \mathrm{H}}$ had no detectable influence on channel conductance in the presence of WT LRRC8A (data not shown). Altogether, these data led us to reason that $L R R C 8 A^{\mathrm{R} 545 \mathrm{H}}$ is a hypomorphic rather than a dominant-negative mutation. 
A

$\begin{array}{cccc}\text { LRR6 } & \text { LRR7 } & \text { LRR8 } & \text { LRR9 } \\ \text { zebrafish } & \text {...LTSLHIKFTDIKEIPLWIYSLKNLSELHLTGNLSAENNRFIVIDGLRELKRLKVLRLKSNLTKLPOVVTDVGVHLOKLSINNEGTKLMVLNSLKKMVN } & 584\end{array}$

LRR7

LRR8

$\begin{array}{cccc}\text { LRR6 } & \text { LRR7 } & \text { LRR8 } & \text { LRR9 } \\ \text { zebrafish } & \text {...LTSLHIKFTDIKEIPLWIYSLKNLSELHLTGNLSAENNRFIVIDGLRELKRLKVLRLKSNLTKLPOVVTDVGVHLOKLSINNEGTKLMVLNSLKKMVN } & 584\end{array}$ Xenopus …LKSLHIKFTDIKEIPLWIYSLKNLEELHLTGNLSAENNRYIVIDGLRELKRLKVLRLKSNLTKLPOVVTDVGVHLQKLSVNNEGTKLMVLNSLKKMVN 598 Human $\quad \ldots$ LRALHIKFTDIKEIPLWIYSLKTLEELHLTGNLSAENNRYIVIDGLRELKRLKVLRLKSNLSKLPQVVTDVGVHLQKLSINNEGTKLIVLNSLKKMAN 599 Mouse $\quad$...LRALHIKFTDIKEIPLWIYSLKTLEELHLTGNLSAENNRYIVIDGLRELKRLKVLRLKSNLSKLPOVVTDVGVHLOKLSINNEGTKLIVLNSLKKMVN 599 $: * * * * * * * * * * * * * * * * * * * . * . * * * * * * * * * * * * * *: * * * * * * * * * * * * * * * * * * * * *: * * * * * * * * * * * * * * * * *: * * * * * * *: * * * * * * * * . * *$

\section{$W T \mid \begin{aligned} & \text { cDNA (Human) } \\ & \text { cDNA (Mouse) } \\ & \text { Protein }\end{aligned}$}

\#90 Human patient $\mid \begin{aligned} & \text { CDNA } \\ & \text { Protein }\end{aligned}$

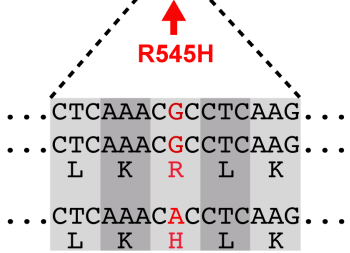

C
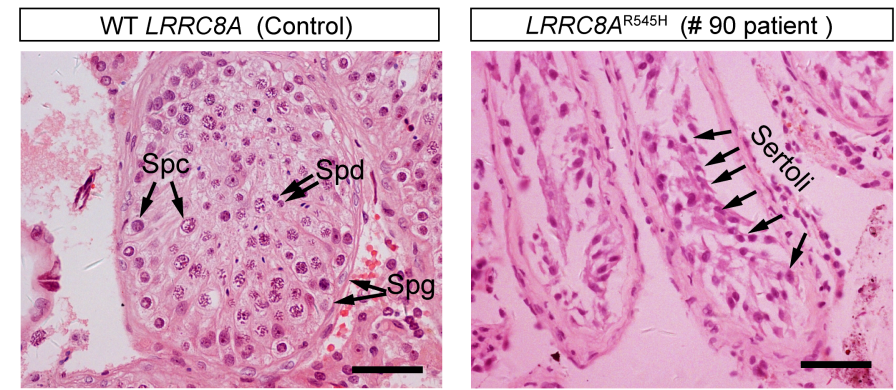

Chr9:g.131671077G>A

D
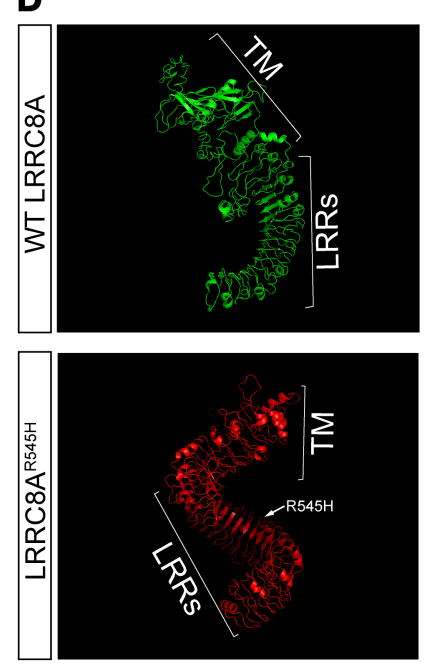

E
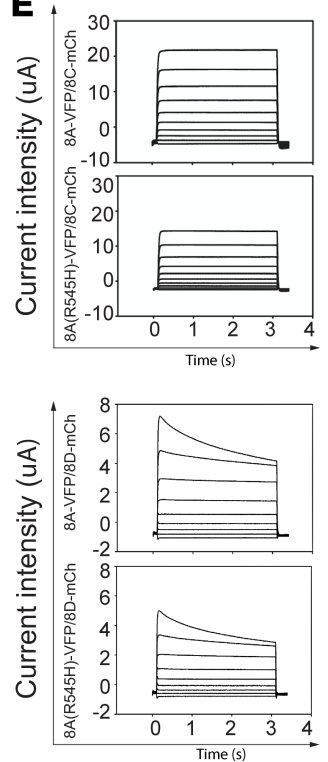

$\mathbf{F}$

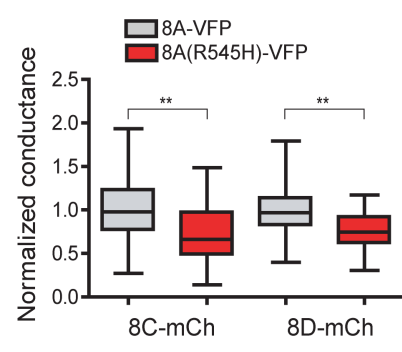

G

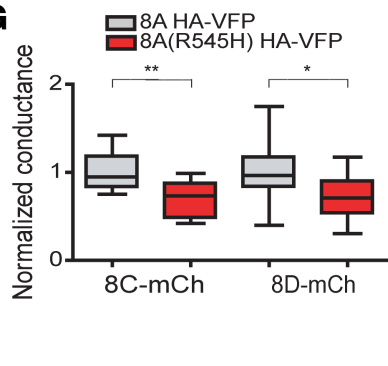

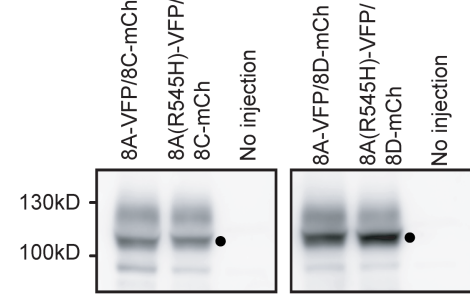

Tubulin $=$

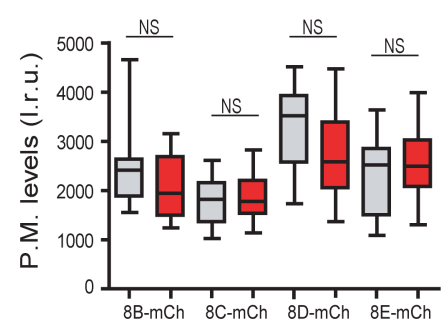

$\square$ 8A HA-VFP

$8 \mathrm{~A}(\mathrm{R} 545 \mathrm{H}) \mathrm{HA}-\mathrm{VFP}$

Figure 6. A potentially hypomorphic mutation (c.1634G>A, R545H) in the LRRC8A gene identified in a male sterile patient with Sertoli cell-only syndrome (SCOS). (A) Whole exome sequencing (WES) identified a missense point mutation (c.1634G>A, p.Arg545His) in a highly conserved region of LRRC8A (top), resulting in the substitution of arginine (R) to histidine (H) (LRRC8A ${ }^{R 545 H}$ ) (bottom) in a male patient suffering from infertility. (B) Sanger resequencing validated the $\mathrm{c} .1634 \mathrm{G}>\mathrm{A}$ mutation in this patient. A red rectangle highlights the mutation site. (C) H\&E staining of testis biopsies from the patient (patient 90) and a fertile control. Spg, spermatogonia; Spc, spermatocyte; Spd, spermatid. Scale bar: $15 \mu \mathrm{m}$. (D) Three-dimensional homology modeling of protein structure for WT (top) and mutant LRRC8 $A^{R 545 H}$ protein (bottom). Note that the R545H mutation altered not only the distribution of LRR domains, but also the topology of the transmembrane structure. The structural prediction was first performed via the Swiss-model server (https://swissmodel.expasy.org/) based on homology modeling data deposited in the protein data bank (PDB). Data were compiled and visualized using PyMOL (www.pymol.org). TM, transmembrane domain; LRRs, leucine-rich repeats. (E-G) In vitro current recordings in the Xenopus oocyte injection system. In vitro-transcribed cRNAs from fluorescently tagged LRRC8 family members as indicated were coinjected into Xenopus oocytes.

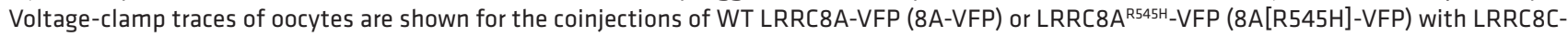
$m$ Cherry $(8 C-m C h)$ or LRRC8D-mCherry (8D-mCh) as labeled $(E)(n=30)$. Comparison of the normalized slope conductance induced by coexpressing $8 \mathrm{~A}-\mathrm{VFP}$ and $8 \mathrm{~A}(\mathrm{R} 545 \mathrm{H})$-VFP with 8C-mCherry or 8D-mCherry as indicated (F, left panel). Three batches of cRNAs were injected into 15 oocytes for each group in each assay. Representative Western blot performed using the same injected oocytes to demonstrate comparable expression levels of the injected cRNAs (F, right panel). Black dots indicate the expected size of fluorescently tagged WT or mutant LRRC8A. Comparison of the normalized slope conductance induced by coexpressing 8A HA-VFP and 8A(R545H) HA-VFP with 8C-mCherry or 8D-mCherry as indicated (G, left panel). ELISAbased luminescence assays utilizing the HA tag illustrated comparable expression of the injected, temdomly tagged cRNAs in the oocyte membranes (G, right panel). Three batches of cRNAs were injected into 15 oocytes for each group in each assay. PM, plasma membrane expression. Data represent mean $\pm \mathrm{SD}$ of 3 independent experiments with 3 batches of cRNA preparations. ${ }^{*} P<0.05,{ }^{*} P<0.01$ (Student's $t$ test). 
As discussed, VRACs in germ cells comprise heteromers of LRRC8A and -B/-C/-D subunits. Due to a lack of detectable current when using fluorescently tagged LRRC8B, we were unable to test the effect of the $L R R C 8 A^{\mathrm{R} 545 \mathrm{H}}$ mutation in the presence of LRRC8B. Thus, testing the complete combinatorial effect of LRRC8 $\mathrm{A}^{\mathrm{R} 545 \mathrm{~A}},-\mathrm{B},-\mathrm{C}$, and $-\mathrm{D}$ was not possible in our oocyte expression system. However, taking into account the evidence that $L R R C 8 A^{\mathrm{R} 545 \mathrm{H}}$ mutation reduced VRAC conductance for both LRR8A/C and LRR8A/D by about $30 \%$, it is reasonable to speculate that the true reduction of VRAC currents due to $L R R C 8 A^{\mathrm{R} 545 \mathrm{H}}$ mutation in a single cell could exceed $60 \%$. We therefore concluded that $L R R C 8 A^{\mathrm{R} 545 \mathrm{H}}$ is a hypomorphic mutation that contributes to the SCOS phenotype in the patient who harbors this mutation.

\section{Discussion}

Volume regulation is a fundamental property of all living cells and is tightly controlled in response to osmotic imbalance. Anion channels are implicated in sperm volume regulation, of which the molecular identity remains poorly understood due to the lack of channel-specific blockers. The recent discovery of LRRC8A as an obligatory subunit of VRAC has invoked extensive follow-up studies, demonstrating multiple functions of LRRC8A-dependent VRAC pathway $(5,7,18,20,23,24)$. However, these studies have mostly been performed in immortalized cell lines, in which cells were stressed with large hypotonic imbalances. The physiological roles of LRRC8A and the importance of LRRC8A-dependent VRAC activity in vivo remain largely unknown. In this study, by utilizing a spontaneous mouse mutation $\left(\mathrm{F} 443^{*}\right)$ and 2 GEMMs, we provided genetic evidence showing that VRAC function in male germline is dependent on LRRC8A, and VRAC activity is essential for volume regulation at different stages of germ cell development in a germ cell-autonomous manner. Germ cells deficient for Lrrc8a progressively degenerate during postnatal development, resulting in an SCOS-like phenotype, consistent with the notion that male germ cells are more vulnerable to volume disturbances than somatic cells (i.e., Sertoli cells) inside the seminiferous tubules.

Strikingly, we identified a putative hypomorphic mutation ( $\left.L R R C 8 A^{R 545 H}\right)$ that is linked to the SCOS phenotype in a sterile male patient. We provided in vitro evidence that LRRC8A ${ }^{\mathrm{R} 545 \mathrm{H}}$ significantly reduced VRAC ion channel activity when reconstituted with LRRC8C or LRRC8D in a Xenopus oocyte expression system. Given that heterozygous $\operatorname{Lrrc}_{3} a^{+/-}(\mathrm{KO})$ and $\operatorname{Lrrc} 8 a^{+/ F 443^{*}}$ (ebo) mice are morphologically indistinguishable from their WT littermates, it is unlikely that the SCOS phenotype associated with the $L R R C 8 A^{R 545 H}$ heterozygous mutation is due to haploinsufficiency, although we cannot rule out the possibility that the functional significance of $L R R C 8 A$ gene differs between mouse and human species. A more likely possibility is that the mutant LRRC8 $\mathrm{A}^{\mathrm{R} 545 \mathrm{H}}$ protein interrupted the functional integrity of the LRRC8 heteromers (LRRC8A-D) that constitute the VRAC channel in germ cells of heterozygous LRRC $8 \mathrm{~A}^{\mathrm{R} 545 \mathrm{H}}$ patient. In contrast, the absence of a phenotypic defect in both heterozygous $L r r c 8 a^{+/-}$and $L r r c 8 a^{+/ F 443^{*}}$ mice could be because the heterozygous KO mice still produce WT protein and the heterozygous $\operatorname{Lrc} 8 a^{F 443^{*}}$ mutants produce the WT plus a truncated LRRC8A protein that, if present, lost the functional domains that could interfere with WT LRRC8A protein and/or other VRAC-regulating LRRC8 subunits. Although the Xenopus oocyte expression system is a robust, valid approach, which consistently recapitulates most of the biophysical and cell biological properties of VRAC (42), we realized that the evidence we provided here for the decreased VRAC activity impacted by $\mathrm{R} 545 \mathrm{H}$ mutation are in vitro data and not thorough. Therefore, further studies (e.g., generation of a mouse knock-in $[\mathrm{KI}]$ model that mimics the $L R R C 8 A^{\mathrm{R} 545 \mathrm{H}}$ allele and crystal structural work) are required to fully elucidate how the mutant LRRC8A ${ }^{\mathrm{R} 545 \mathrm{H}}$ protein impairs VRAC channel activity, leading to the SCOS phenotype.

Previous studies, using electrophysiological patch-clamp recordings, have identified a handful of ion channels that are either ubiquitously expressed or are sperm specific, such as SLO3 (pH-regulated $\mathrm{K}^{+}$channel) and CatSper ( $\mathrm{Ca}^{2+}$ permeable cation channel) $(3,10,43-45)$, in the testis. Genetic evidence demonstrates that these channels, which are relatively well characterized in terms of their biophysical properties and functional importance, play versatile roles in sperm capacitation and motility, the acrosome reaction, and fertilization $(3,4,43,46)$. In contrast, the precise molecular make-up and the physiological function of VRAC in the germline are not understood. Several lines of evidence suggest that sperm volume regulation must be tightly controlled during sperm transition through the rete testis, efferent ductules, caput, and cauda of the epididymis and into the female reproductive tract. First, 3 GEMMs, c-Ros (Ros1) KO, Gpx5-Tag2, and Esr1 KO (12, 47,48 ), in which the manipulated genes compromised epithelial function causing reduced fluid osmolality in the initial segment of caput epididymis or in the efferent duct, were unexpectedly found to be infertile due 
to swollen sperm. Second, Bestrophin 1 (BEST1), a controversial $\mathrm{Ca}^{2+}$-activated $\mathrm{Cl}^{-}$channel linked to $\mathrm{Ca}^{2+}$ homeostasis and neurotransmitter release, was recently discovered to specifically regulate sperm volume, not $\mathrm{Ca}^{2+}$ homeostasis, to cope with hypotonicity $(49,50)$. BEST1 protein is specifically localized to the equatorial segment of the sperm head, and Best1-KO mice showed severe subfertility, owing to compromised RVD ability causing sperm swelling (49-51). Third, the dedicated water-permeable channel aquaporin 3 (AQP3) has been validated as a bona fide sperm intrinsic volume-regulatory player during RVD upon sperm swelling. AQP3 is exclusively localized in the principal piece of the sperm tail, and Aqp3-null mouse sperm are characterized by curly or angulated tails (52). The GEMM evidence unambiguously proved that the volume-regulatory capacity is an important determinant of the fertilizing potential of sperm. However, neither Best1-nor Aqp3-deficient male mice are completely infertile, suggesting the existence of other possible RVD-mediating machinery in germ cells. Moreover, previous studies focused primarily on mature sperm (i.e., after their exit from seminiferous tubules). The importance of RVD in germ cell development during spermatogenesis in seminiferous tubules is currently unclear. We showed that loss of LRRC8A-dependent VRAC activity leads to a disruption in the development of immature germ cells, including spermatogonia, spermatocytes, and spermatids, and these defective germ cells progressively degenerate over time, in the $\operatorname{Lrc} 8 a^{\mathrm{F} 443^{*} / \mathrm{F} 443^{*}}, \operatorname{Lrrc} 8 a-\mathrm{KO}$, and $L r r c 8 a$-cKO testis. Indeed, we observed large vacuoles in male mice that reached 12 months of age due to a massive loss of germ cells in the Stra8-Cre driven cKO testis, which resembles the human SCOS phenotype. These mouse models provide genetic evidence that volume-regulatory activity is essential for spermatogenesis in the testis, as well as sperm maturation in the epididymis.

Apoptotic cells initiate persistent cell shrinkage preceding caspase activation and DNA fragmentation in a process known as apoptotic volume decrease (AVD), which is thought to share the same $\mathrm{Cl}^{-}$ channel activity as VRAC (53). Germ cells are highly susceptible to apoptotic stimuli linked to the inherent genome-wide chromatin remodeling. Surprisingly, we didn't see an increase in apoptosis in germ cells from $\operatorname{Lrc} 8 a^{\mathrm{F} 443^{*} / \mathrm{F} 443^{*}}$ or $\operatorname{Lrr} 8 \mathrm{a}$-KO mice testis by immunolabeling (data not shown). Consistently, genome-wide RNA-seq analyses identified overrepresented Gene Ontology (GO) terms linked to disruptions in development and reproductive system metabolism, but not apoptosis (Supplemental Figure 3, A and B). Likewise, Gene Set Enrichment Analysis (GSEA) identified only metabolic pathways unrelated to apoptosis (Supplemental Figure 3, A-C). Such evidence suggests that the mutant germ cells died through an apoptosis-independent pathway (e.g., in extreme cases, cell bursting). This is in agreement with the notion that LRRC8A-depedent VRAC is important for AVD and that loss of LRRC8A function impedes apoptosis (53). Given that all members of the LRRC8 family except LRRC8E are present in germ cells (Figure 1), and that the precise combination of LRRC8 heteromers determines the substrate selectivity of a particular $\operatorname{VRAC}(5,21,26)$, it is conceivable that VRACs in germ cells might have other functions in addition to volume regulation, such as the uptake of specific paracrine factors secreted by the neighboring Sertoli cells.

It is interesting to note that both male and female $\operatorname{Lrrc8} a^{\mathrm{F} 443^{*} / \mathrm{F} 443^{*}}$ and $\operatorname{Lrr} 8 \mathrm{a}-\mathrm{KO}$ mice are completely infertile. However, in contrast to the defects in spermatogenesis we observed in males, oogenesis was normal in females, suggesting that LRRC8A and LRRC8A-dependent VRAC activity are dispensable for oogenesis. This supports the notion that cell volume regulation is not critical for oocyte growth in the follicles (54). Since every growing oocyte is encompassed by a specialized extracellular matrix shell zona pellucida (ZP), oocyte volume is essentially determined by its adhesion to the ZP (54). Nonetheless, embryos derived from LRRC8A-deficient oocytes exhibit early developmental arrest and progressive degeneration, suggesting that functional integrity of VRAC in oocytes is critical for preimplantation embryonic development.

In summary, we showed that LRRC8A-mediated VRAC is a key volume regulator, essential for male germ cell development. Genetic mutations that diminish LRRC8A-dependent VRAC activity may lead to germ cell defects, causing infertility in both mice and humans. Hence, LRRC8A could potentially serve as a diagnostic or therapeutic target for human patients suffering from infertility.

\section{Methods}

Supplemental Methods are available online with this article.

Mice, targeted region sequencing, and genotyping. The $L r r c 8 a^{\text {ebo }}$ autosomal recessive mutation arose spontaneously in a substrain of BALB/c mice from the Institut Pasteur. Coisogenic mice harboring the mutation were transferred to MD Anderson, where the mutant allele was introgressed into the FVB/N background by classical and marker-assisted backcrossing to produce the FVB/N.Cg-Lrrc $8 a^{e b o}$ congenic strain (>N10). 
Microsatellite genotyping (genome scan using polymorphic markers) was carried out by Laboratory Animal Genetic Services located at MD Anderson. Specific pathogen-free (SPF) FVB/N.Cg-Lrrc $8 a^{\text {ebo }}$ mice were obtained through rederivation by embryo transfer and used for the phenotypic characterization. For the targeted region sequencing, targeted DNA for the candidate region on chromosome 2 was sequenced at the MD Anderson Sequencing and Microarray Facility using a mouse capture array from Ambry Genetics Corp. and according to the manufacturer's protocol. For genotyping, in order to distinguish the 2-bp deletion difference between the WT and $\operatorname{Lrc} 8 a^{F 443^{*}}$ alleles, 2 specific forward primers tagged with the fluorescent dyes VIC and 6-FAM were designed against the WT and $L r r c 8 a^{F 443^{*}}$ alleles, respectively, along with a common reverse primer. A standard endpoint PCR assay was performed according to the Kompetitive Allele Specific PCR genotyping system (KASP) (LGC Genomics) protocol. Fluorescent intensity was recorded using the Fluostar Optima plate reader (BMG Labtech).

To generate the constitutive Lrrc8a KO mice (KO), sperm from C57BL/6N-Lrrc8 $a^{\text {tm1(KOMP)Vlcg }}{ }^{\text {null/Lac- }}$ Zreporter line were purchased from the Knockout Mouse Project (KOMP) repository (UCD, Davis, California, USA), and live pups were recovered through in vitro fertilization (IVF). To generate the germlinespecific Lrrc8a-cKO mouse model, sperm purchased from KOMP (C57BL/6N-Lrrc8 $a^{\text {tm1.1(KOMP)Vlcg }}$, in which 2 LoxP sites flank the exon3) were used to produce $L r r c 8 a^{f / f l}$ mice through IVF. Stra8-Cre deletor mice (C57BL/6J, The Jackson Laboratory, Stock No. 008208) were intercrossed with $L r r c 8 a^{f l / f l}$ mice to generate the Stra8-Cre; Lrrc8 ${ }^{f l / f l}$ (cKO) mice.

Human samples and WES. A total of 102 patients diagnosed with SCOS were recruited by the reproductive center at the University of Science and Technology of China. The diagnostic criteria for SCOS are as follows: (a) no sired children and no visible sperm discovered in the pellets of semen samples taken on 3 different occasions; (2) no obstruction, inflammation, or injury in the reproductive system or pelvic cavity; (c) no karyotypic aberrations or Y chromosome microdeletions; and (d) only Sertoli cells are present in the seminiferous tubules. Samples from an in-house cohort of healthy, fertile males (254 healthy Han Chinese males and 283 healthy European-descent males) were used as controls.

WES was performed using genomic DNA isolated from whole peripheral blood. The fragments of human whole exome were captured using the Agilent Human All Exon $50 \mathrm{Mb}$ kit (Agilent Technology) and sequenced on a Illumina HiSeq2000. Clean reads were mapped to the human reference genome (hg19) using the Burrows-Wheeler Alignment tool (http://bio-bwa.sourceforge.net/). To identify detrimental genetic mutations, variants fulfilling the following criteria were called and annotated using the Genome Analysis Toolkit and ANNOVAR respectively: (a) nonsense, missense, frameshift, or splicing variants; (b) absent or rare in dbSNP (http://www.ncbi.nlm.nih.gov/snp/), the 1000 Genomes; and (c) our in-house WES database of 254 healthy, fertile Han Chinese males and 283 healthy, fertile European-descent males.

Histopathology. Freshly dissected testes samples were immediately fixed in Bouin's solution for 24 hours at room temperature. Other fresh tissues were fixed in the $10 \%$ formalin solution for 48 hours at room temperature, unless otherwise stated. Following fixation, tissues were washed in $70 \%$ ethyl alcohol- $\mathrm{Li}_{2} \mathrm{CO}_{3}-$ saturated solution and embedded in paraffin for histological analysis. Testicular cross sections (5 $\mu \mathrm{m}$ thickness) were evaluated by using either periodic acid-Schiff (PAS) or H\&E staining.

To analyze the sperm parameters, mice were sacrificed through $\mathrm{CO}_{2}$ inhalation followed by cervical dislocation. Fresh cauda epididymis was dissected, and sperm were released into TYH medium at specific osmolalities (approximately 290, 330, 360, and $410 \mathrm{mOsm} / \mathrm{kg}$ ) through puncturing the cauda epididymis using 2 fine needles. Sperm morphology and motility were examined using phase-contrast microscopy and a computer-assisted sperm analysis (CASA) station, respectively.

Ovary follicles in the cross-sections of paraffin-embedded slides were identified as primordial, primary, secondary, antral, or corpus luteum (CL) based on cellular composition and morphology. Atretic follicles, as characterized by pyknotic granulosa cells and/or a grossly misshapen oocyte, were omitted from counts. Sections were then combined in the third dimension (stacked, $600 \mu \mathrm{m}$ total thickness) to analyze the average total number of each follicular subtype in each sample. Statistical comparisons were carried out using at least 3 females for each genotype. Samples in the slides were scanned and digitalized using an Aperio ScanScope CS slide scanner (Aperio Technologies).

IHC. Monoclonal LRRC8A antibody (clone 8H9) was purchased from MilliporeSigma. For immunolabeling, testicular samples were fixed in $4 \%$ paraformaldehyde (PFA) at $4^{\circ} \mathrm{C}$ overnight, followed by washing in $10 \%-30 \%$ sucrose for 3 hours at room temperature. Cryo-preserved testes were cut into cross-sections $(5 \mu \mathrm{m})$, mounted on Superfrost Plus glass slides, and air-dried. Samples were subject to antigen retrieval 
processing by submerging the slides in boiling citrate buffer ( $\mathrm{pH}$ 6.0) for 15 minutes. After 3 washes in $1 \times$ PBS, samples were incubated in blocking buffer (5\%BSA + 0.3\% Triton X-100) at room temperature for 1 hour, followed by incubation with primary antibody at $4^{\circ} \mathrm{C}$, overnight. The next day, slides were washed 3 times in $1 \times$ PBS and incubated with secondary antibody conjugated with a specific fluorescent dye for 1 hour at room temperature. After a brief washing in $1 \times$ PBS, slides were mounted using ProLong Gold Antifade Mountant with DAPI medium (Thermo Fisher Scientific). Samples were imaged using a Zeiss LSM880 Confocal system.

To determine the subcellular localization of LRRC8A protein in germ cells, squashed seminiferous tubules were generated following published procedures (55). For immunolabeling of paraffin-embedded samples, sections were cleared with xylenes and rehydrated in a gradient of alcohol, followed by incubation with $3 \% \mathrm{H}_{2} \mathrm{O}_{2}$ to block endogenous peroxidase activity. After incubation with blocking buffer (10\% horse serum) for 1 hour at room temperature, sections were incubated with primary antibody for 1 hour at room temperature or at $4^{\circ} \mathrm{C}$ overnight. Immunodetection was performed using a VectaStain ABC kit (Vector Laboratories) and 3,3'-diaminobenzidine substrate (Vector Laboratories) following standard procedures.

Western blot. Total protein preparation from mice testes for Western blot analysis has been described in detail (56). Protein lysates were prepared by homogenizing the snap-frozen tissues in the radioimmunoprecipitation assay (RIPA) buffer supplemented with the proteinase inhibitors (Roche Diagnostics). Protein concentrations were determined by the Bradford assay according to the manufacturer's protocol (Bio-Rad). A total of $40 \mu \mathrm{g}$ protein was separated on an 11\% SDS-PAGE gel and was transferred to PVDF membrane for each sample. Blots were treated with ECL reagent (Amersham Bioscicence) and exposed to X-ray film. Actin served as a loading control.

Electron microscopy. Freshly dissected testes were carefully cut into small pieces and fixed at $4^{\circ} \mathrm{C}$ for 2 days in buffer containing 3\% paraformaldehyde, $3 \%$ glutaraldehyde, and $0.2 \%$ picric acid (Electron Microscopy Sciences). Sperm released from the cauda epididymis were fixed under the same conditions. Fixed samples were sent to the High Resolution Electron Microscopy Facility (HREMF) at MD Anderson Cancer Center for further processing following standard protocols as described previously. Electron micrographs were obtained using a JEM1010 TEM and a JSM 5900 scanning electron microscope. To calculate the NC ratio, the cross-section areas of the nucleus (An) and the cell (Ac) were measured from 2-dimensional TEM images using Image $(\mathrm{NIH})$. The NC ratio was estimated using the formula below (57):

$V_{n} / V_{c}=\left(A_{n} / A_{c}\right)^{3 / 2}$

where $\mathrm{Vn}$ represents volume of nucleus and $\mathrm{Vc}$ represents volume of cell.

The experiments were performed in biological triplicates, and the statistics were calculated from 20 cells from each sample.

Electrophysiological recordings. Testicular single cell suspensions were prepared as described previously with minor modifications (36). Briefly, the interstitial cells between the seminiferous tubules were removed by sedimentation following collagenase IV digestion. Single cell suspensions were achieved by trypsin digestion. Dissociated germ cells were allowed to attach to cover-glasses precoated with poly-D-lysine and laminin in 35-mm culture dishes containing 90\% DMEM and 10\% newborn calf serum. The cells were incubated in a $\mathrm{CO}_{2}$ incubator $\left(5 \% \mathrm{CO}_{2}\right)$ at $37^{\circ} \mathrm{C}$ for $1-4$ hours before recording. Different types of germ cells (spermatogonia, spermatocytes, and round spermatids) were identified by cell size and morphology using differential interference contrast microscopy. Whole-cell patch-clamp recordings were performed with an EPC-10 amplifier (HEKA Instruments, Lambrecht, Germany). Recording electrodes (resistance, 2-3 M 2 ) were pulled from thick-walled borosilicate glasses and fire-polished. After a whole-cell configuration was established, the cell membrane capacitance and series resistance were electronically compensated. The signals were digitized at $10 \mathrm{kHz}$ and low-pass filtered at $1 \mathrm{kHz}$. All recordings were performed at $25^{\circ} \mathrm{C}$. External hypotonic solution $(220 \mathrm{mOsm} / \mathrm{kg}$ ) was composed of $95 \mathrm{mM} \mathrm{NaCl}, 2 \mathrm{mM} \mathrm{CaCl}, 1$ $\mathrm{mM} \mathrm{MgCl}, 5 \mathrm{mM} \mathrm{CsCl}, 10 \mathrm{mM}$ HEPES, and $10 \mathrm{mM}$ glucose ( $\mathrm{pH} 7.4$ adjusted with $\mathrm{NaOH}$ ). Sucrose was then added to make normotonic solution $(320 \mathrm{mOsm} / \mathrm{kg})$. The internal pipette solution consisted of 120 mM Cs-aspartate, $10 \mathrm{mM} \mathrm{Cs}_{4}$ BAPTA, $4 \mathrm{mM} \mathrm{MgATP}, 2 \mathrm{mM} \mathrm{MgCl}_{2}, 8 \mathrm{mM} \mathrm{NaCl}$, and $10 \mathrm{mM}$ HEPES ( $\mathrm{pH} 7.2$ adjusted with $\mathrm{CsOH}$ ). To record VRAC currents in hypotonic solution, cells were held at -70 $\mathrm{mV}$ with voltage ramps from $-100 \mathrm{mV}$ to $+100 \mathrm{mV}$ over $400 \mathrm{~ms}$ every 5 seconds. Currents were recorded for 5 minutes after bath application of hypotonic solution, and those currents measured at $-80 \mathrm{mV}$ and $+80 \mathrm{mV}$ were plotted to show the time course of current changes. In addition, currents were recorded 
using voltage steps from $-70 \mathrm{mV}$ to $+140 \mathrm{mV}$ at $10-\mathrm{mV}$ intervals (step duration, $40 \mathrm{~ms}$ ) 5 minutes after bath application of hypotonic solution. VRAC current density was calculated by normalizing the current to capacitance to account for differences in cell sizes. To determine the reversal potential of currents, an equimolar concentration of NMDG- $\mathrm{Nl}$ was used to replace $\mathrm{NaCl}, \mathrm{KCl}$, or $\mathrm{CaCl}_{2}$, and $\mathrm{Cl}^{-}$was replaced with aspartate when needed. The permeable cation-free solutions contained (in mM) 100 NMDG-Cl, 10 HEPES, 10 glucose, and $2 \mathrm{Na}_{2}$-ATP at $\mathrm{pH}$ 7.4. Data analysis was performed using the Pulse software (HEKA Instruments).

Xenopus oocytes injection and voltage-clamp recordings. All procedures for plasmids construction, Xenopus oocyte preparation, injection, and voltage-clamp recordings were performed as described in detail previously $(41,58)$. Briefly, fluorescently tagged plasmids were linearized by NotI digestion prior to in vitro transcription using the mMessage mMachine SP6 kit (Ambion). Human $L R R C 8 A^{\mathrm{R} 545 \mathrm{H}}$ variant was generated by site-directed mutagenesis. Electrophysiology was performed in the hypotonic solution containing (in $\mathrm{mM}$ ) $48 \mathrm{NaCl}, 2 \mathrm{KCl}, 1.8 \mathrm{CaCl}_{2}, 1 \mathrm{MgCl}_{2}$, and 10 Hepes with pH 7.3 (120 mOsm/kg). cRNA (50 nl) containing $10 \mathrm{ng}$ of WT LRRC8A-VFP or LRRC8A(R545H)-VFP were coexpressed with $10 \mathrm{ng}$ of LRRC8C-mCh or LRRC8D-mCh. For the coexpression assays, $5 \mathrm{ng}$ of WT LRRC8A-VFP plus 5 ng of LRRC8A ${ }^{\text {R545H.VFP }}$ were coexpressed together with $10 \mathrm{ng}$ of LRRC8C-mCh or LRRC8D-mCh. To measure the protein expression levels in the membrane for surface analysis, HA-tagged WT LRRC8A plasmid (LRRC8A HA-VFP) and mutant (LRRC8A[R545] HA-VFP) were generated by inserting an HA epitope into the first extracellular loop of LRRC8A. For each plasmid, 3 batches of cRNAs were prepared, and each assay was carried out as described, with the oocyte number indicated. To estimate the slope conductance, the Axon pClamp Clampfit software was used. Slope conductance was calculated by fitting "straight line, standard" in a +10 to $+70 \mathrm{mV}$ voltage range from the I-V curves. In order to unify and clarify the experiments, results were normalized to the WT values in each of the combinations tested.

Statistics. All experiments were performed using at least in 3 biological replicates, unless otherwise stated. Data were presented as mean $\pm \mathrm{SD}$, unless indicated. Statistical values were calculated using oneway Student's $t$ test between groups or one-way ANOVA as indicated. $P$ values less than 0.05 were deemed as statistically significant.

Study approval. All animal care and use procedures were approved by the IACUC of the MD Anderson Cancer Center and were in accordance with the NIH regulations for the care and use of animals in research.

\section{Author contributions}

FB and JB conceived and designed the study, conducted and analyzed the majority of experiments, and prepared the manuscript with input from TC, MTB, SYRD, JHR, RE, HLP, RSG, and QS. CJP, JK, HZ, CJM, TH, JJ, CDP, JC, MD, MHZ, YH, and HGP performed some experiments and analyzed data. JLG, RSG, RE, and HLP provided scientific insight, supervised some analyses, and edited the manuscript. KL and YL conducted the bioinformatics analyses.

\section{Acknowledgments}

We thank the Research Animal Support Facility-Smithville for their assistance with the maintenance of the mouse strains, the Histology Core for processing the samples, the Molecular Biology Facility Core for RNA sequencing, the Bioinformatics and Biostatistics Services for data analysis, and Briana Dennehey for editing the manuscript. This study made use of the Research Animal Support Facility-Smithville (including Laboratory Animal Genetic Services and Research Histology Pathology and Imaging Core), the Sequencing and Microarray Facility, and the HREMF, all of which are supported by P30 CA016672 DHHS/ NCI Cancer Center Support Grant to MD Anderson Cancer Center. This work was supported in part by Departmental Funds and Molecular Biology Facility Core supported by CPRIT grant RP170002 to Dr. Jianjun Shen, National Basic Research Program of China (2014CB943101) and the National Natural Science Foundation of China (31371519 and 313111245) to QS, and R01 ES016591 to JHR.

Address correspondence to: Fernando Benavides or Jianqiang Bao, The University of Texas, MD Anderson Cancer Center, Department of Epigenetics and Molecular Carcinogenesis, 1808 Park Road 1C, P.O. Box 389, Smithville, Texas 78957, USA. Phone: 512.237.9343 Email: fbenavid@mdanderson.org (F. Benavides). Phone: 512.237.6613; Email: jqbao@ustc.edu.cn (J. Bao). 
1. Hoffmann EK, Lambert IH, Pedersen SF. Physiology of cell volume regulation in vertebrates. Physiol Rev. 2009;89(1):193-277.

2. Day RE, et al. Human aquaporins: regulators of transcellular water flow. Biochim Biophys Acta. 2014;1840(5):1492-1506.

3. Yeung CH, Barfield JP, Cooper TG. Chloride channels in physiological volume regulation of human spermatozoa. Biol Reprod. 2005;73(5):1057-1063.

4. Cooper TG, Yeung CH. Involvement of potassium and chloride channels and other transporters in volume regulation by spermatozoa. Curr Pharm Des. 2007;13(31):3222-3230.

5. Jentsch TJ, Lutter D, Planells-Cases R, Ullrich F, Voss FK. VRAC: molecular identification as LRRC8 heteromers with differential functions. Pflugers Arch. 2016;468(3):385-393.

6. Pedersen SF, Klausen TK, Nilius B. The identification of a volume-regulated anion channel: an amazing Odyssey. Acta Physiol (Oxf). 2015;213(4):868-881.

7. Syeda R, et al. LRRC8 Proteins Form Volume-Regulated Anion Channels that Sense Ionic Strength. Cell. 2016;164(3):499-511.

8. Mruk DD, Cheng CY. Tight junctions in the testis: new perspectives. Philos Trans R Soc Lond, B, Biol Sci. 2010;365(1546):16211635 .

9. Cooper TG, et al. Mouse models of infertility due to swollen spermatozoa. Mol Cell Endocrinol. 2004;216(1-2):55-63.

10. Petrunkina AM, Harrison RA, Ekhlasi-Hundrieser M, Töpfer-Petersen E. Role of volume-stimulated osmolyte and anion channels in volume regulation by mammalian sperm. Mol Hum Reprod. 2004;10(11):815-823.

11. Yeung CH, Barfield JP, Cooper TG. Physiological volume regulation by spermatozoa. Mol Cell Endocrinol. 2006;250(1-2):98-105.

12. Yeung $\mathrm{CH}$, et al. Sperm volume regulation: maturational changes in fertile and infertile transgenic mice and association with kinematics and tail angulation. Biol Reprod. 2002;67(1):269-275.

13. Domeniconi RF, Souza AC, Xu B, Washington AM, Hinton BT. Is the Epididymis a Series of Organs Placed Side By Side? Biol Reprod. 2016;95(1):10.

14. Yeung CH, Callies C, Rojek A, Nielsen S, Cooper TG. Aquaporin isoforms involved in physiological volume regulation of murine spermatozoa. Biol Reprod. 2009;80(2):350-357.

15. Smits G, Kajava AV. LRRC8 extracellular domain is composed of 17 leucine-rich repeats. Mol Immunol. 2004;41(5):561-562

16. Abascal F, Zardoya R. LRRC8 proteins share a common ancestor with pannexins, and may form hexameric channels involved in cell-cell communication. Bioessays. 2012;34(7):551-560.

17. Sawada A, et al. A congenital mutation of the novel gene LRRC8 causes agammaglobulinemia in humans. JClin Invest. 2003;112(11):1707-1713.

18. Qiu Z, et al. SWELL1, a plasma membrane protein, is an essential component of volume-regulated anion channel. Cell. 2014;157(2):447-458.

19. Voss FK, et al. Identification of LRRC8 heteromers as an essential component of the volume-regulated anion channel VRAC. Science. 2014;344(6184):634-638.

20. Gradogna A, Gavazzo P, Boccaccio A, Pusch M. Subunit-dependent oxidative stress sensitivity of LRRC8 volume-regulated anion channels. J Physiol (Lond). 2017;595(21):6719-6733.

21. Planells-Cases R, et al. Subunit composition of VRAC channels determines substrate specificity and cellular resistance to Ptbased anti-cancer drugs. EMBO J. 2015;34(24):2993-3008.

22. Behe P, et al. The LRRC8A Mediated "Swell Activated" Chloride Conductance Is Dispensable for Vacuolar Homeostasis in Neutrophils. Front Pharmacol. 2017;8:262.

23. Schober AL, Wilson CS, Mongin AA. Molecular composition and heterogeneity of the LRRC8-containing swelling-activated osmolyte channels in primary rat astrocytes. J Physiol (Lond). 2017;595(22):6939-6951.

24. Zhang Y, et al. SWELL1 is a regulator of adipocyte size, insulin signalling and glucose homeostasis. Nat Cell Biol. 2017;19(5):504-517.

25. Lee CC, Freinkman E, Sabatini DM, Ploegh HL. The protein synthesis inhibitor blasticidin s enters mammalian cells via leucine-rich repeat-containing protein 8D. J Biol Chem. 2014;289(24):17124-17131.

26. Lutter D, Ullrich F, Lueck JC, Kempa S, Jentsch TJ. Selective transport of neurotransmitters and modulators by distinct volumeregulated LRRC8 anion channels. J Cell Sci. 2017;130(6):1122-1133.

27. Kang C, et al. SWELL1 is a glucose sensor regulating $\beta$-cell excitability and systemic glycaemia. Nat Commun. 2018 ;9(1):367.

28. Lalouette A, Lablack A, Guenet JL, Montagutelli X, Segretain D. Male sterility caused by sperm cell-specific structural abnormalities in ebouriffé, a new mutation of the house mouse. Biol Reprod. 1996;55(2):355-363.

29. Platt CD, et al. Leucine-rich repeat containing 8A (LRRC8A)-dependent volume-regulated anion channel activity is dispensable for T-cell development and function. J Allergy Clin Immunol. 2017;140(6):1651-1659.e1.

30. Kumar L, et al. Leucine-rich repeat containing 8A (LRRC8A) is essential for T lymphocyte development and function. $J$ Exp Med. 2014;211(5):929-942.

31. GTEx Consortium. Human genomics. The Genotype-Tissue Expression (GTEx) pilot analysis: multitissue gene regulation in humans. Science. 2015;348(6235):648-660.

32. Margolin G, Khil PP, Kim J, Bellani MA, Camerini-Otero RD. Integrated transcriptome analysis of mouse spermatogenesis. BMC Genomics. 2014;15:39.

33. Li W, et al. Chd5 orchestrates chromatin remodelling during sperm development. Nat Commun. 2014;5:3812.

34. Bao J, Rousseaux S, Shen J, Lin K, Lu Y, Bedford MT. The arginine methyltransferase CARM1 represses p300•ACT•CREM $\tau$ activity and is required for spermiogenesis. Nucleic Acids Res. 2018;46(9):4327-4343.

35. Kotaja N, et al. Preparation, isolation and characterization of stage-specific spermatogenic cells for cellular and molecular analysis. Nat Methods. 2004;1(3):249-254.

36. Bao J, et al. UPF2-Dependent Nonsense-Mediated mRNA Decay Pathway Is Essential for Spermatogenesis by Selectively Eliminating Longer 3'UTR Transcripts. PLoS Genet. 2016;12(5):e1005863.

37. Coutton C, Escoffier J, Martinez G, Arnoult C, Ray PF. Teratozoospermia: spotlight on the main genetic actors in the human. Hum Reprod Update. 2015;21(4):455-485.

38. Krausz C, Riera-Escamilla A. Genetics of male infertility. Nat Rev Urol. 2018;15(6):369-384.

39. Dong C, et al. Comparison and integration of deleteriousness prediction methods for nonsynonymous SNVs in whole exome 
sequencing studies. Hum Mol Genet. 2015;24(8):2125-2137.

40. Biasini M, et al. SWISS-MODEL: modelling protein tertiary and quaternary structure using evolutionary information. Nucleic Acids Res. 2014;42(Web Server issue):W252-W258

41. Gaitán-Peñas H, et al. Investigation of LRRC8-Mediated Volume-Regulated Anion Currents in Xenopus Oocytes. Biophys J. 2016;111(7):1429-1443.

42. Gaitán-Peñas H, Pusch M, Estévez R. Expression of LRRC8/VRAC Currents in Xenopus Oocytes: Advantages and Caveats. Int J Mol Sci. 2018;19(3):E719.

43. Yeung $\mathrm{CH}$, Barfield JP, Cooper TG. The role of anion channels and $\mathrm{Ca} 2+$ in addition to $\mathrm{K}+$ channels in the physiological volume regulation of murine spermatozoa. Mol Reprod Dev. 2005;71(3):368-379.

44. Qi H, et al. All four CatSper ion channel proteins are required for male fertility and sperm cell hyperactivated motility. Proc Natl Acad Sci USA. 2007;104(4):1219-1223.

45. Zeng XH, Yang C, Kim ST, Lingle CJ, Xia XM. Deletion of the Slo3 gene abolishes alkalization-activated K+ current in mouse spermatozoa. Proc Natl Acad Sci USA. 2011;108(14):5879-5884.

46. Chan HC, Chen H, Ruan Y, Sun T. Physiology and pathophysiology of the epithelial barrier of the female reproductive tract: role of ion channels. Adv Exp Med Biol. 2012;763:193-217.

47. Yeung CH, Sonnenberg-Riethmacher E, Cooper TG. Infertile spermatozoa of c-ros tyrosine kinase receptor knockout mice show flagellar angulation and maturational defects in cell volume regulatory mechanisms. Biol Reprod. 1999;61(4):1062-1069.

48. Joseph A, Shur BD, Ko C, Chambon P, Hess RA. Epididymal hypo-osmolality induces abnormal sperm morphology and function in the estrogen receptor alpha knockout mouse. Biol Reprod. 2010;82(5):958-967.

49. Fischmeister R, Hartzell HC. Volume sensitivity of the bestrophin family of chloride channels. J Physiol (Lond). 2005;562(Pt 2):477-491.

50. Milenkovic A, et al. Bestrophin 1 is indispensable for volume regulation in human retinal pigment epithelium cells. Proc Natl Acad Sci USA. 2015;112(20):E2630-E2639.

51. Strauß O, Müller C, Reichhart N, Tamm ER, Gomez NM. The role of bestrophin-1 in intracellular Ca(2+) signaling. Adv Exp Med Biol. 2014;801:113-119.

52. Chen $\mathrm{Q}$, et al. Aquaporin3 is a sperm water channel essential for postcopulatory sperm osmoadaptation and migration. Cell Res. 2011;21(6):922-933.

53. Okada Y, Maeno E, Shimizu T, Dezaki K, Wang J, Morishima S. Receptor-mediated control of regulatory volume decrease (RVD) and apoptotic volume decrease (AVD). J Physiol (Lond). 2001;532(Pt 1):3-16.

54. Tartia AP, Rudraraju N, Richards T, Hammer MA, Talbot P, Baltz JM. Cell volume regulation is initiated in mouse oocytes after ovulation. Development. 2009;136(13):2247-2254.

55. Bao J, et al. RANBP17 is localized to the XY body of spermatocytes and interacts with SPEM1 on the manchette of elongating spermatids. Mol Cell Endocrinol. 2011;333(2):134-142

56. Yao PL, Lin YC, Sawhney P, Richburg JH. Transcriptional regulation of FasL expression and participation of sTNF-alpha in response to sertoli cell injury. J Biol Chem. 2007;282(8):5420-5431.

57. Finan JD, Leddy HA, Guilak F. Osmotic stress alters chromatin condensation and nucleocytoplasmic transport. Biochem Biophys Res Commun. 2011;408(2):230-235.

58. Gaitán-Peñas H, et al. Leukoencephalopathy-causing CLCN2 mutations are associated with impaired Cl-channel function and trafficking. J Physiol (Lond). 2017;595(22):6993-7008. 